\title{
Megamaser detection and nuclear obscuration in Seyfert galaxies ${ }^{\star}$
}

\author{
M. Ramolla ${ }^{1}$, M. Haas ${ }^{1}$, V. N. Bennert ${ }^{2}$, and R. Chini ${ }^{1,3}$ \\ 1 Astronomisches Institut, Ruhr-Universität Bochum, Universitätsstraße 150, 44801 Bochum, Germany \\ e-mail: ramolla@astro.rub.de \\ 2 Department of Physics, University of California, Santa Barbara, CA 93106, USA \\ 3 Facultad de Ciencias, Universidad Católica del Norte, Antofagasta, Chile
}

Received 21 June 2010 / Accepted 3 February 2011

\begin{abstract}
We revisit the relation between $\mathrm{H}_{2} \mathrm{O}$ maser detection rate and nuclear obscuration for a sample of 114 Seyfert galaxies drawn from the CfA, $12 \mu \mathrm{m}$, and IRAS F25/F60 catalogs. These sources have mid-infrared spectra from the Spitzer Space Telescope and we search for accompanying X-ray and [O III] $5007 \AA$ fluxes from the literature. We use the strength of the [O IV] $25.9 \mu \mathrm{m}$ emission line as a tracer of the intrinsic AGN strength. After the normalization by [O IV], the observed X-ray flux provides information about $\mathrm{X}$-ray absorption. The distribution of X-ray/[O IV] flux ratios is significantly different for masers and non-masers: The maser detected Seyfert-2s (Sy 1.8-2.0) populate a distinct X-ray/[O IV] range, which is, on average, about a factor of four lower than the range of Seyfert-2 non-masers and about a factor of ten lower than the range of Seyfert-1s (Sy 1.0-1.5). Non-masers are almost equally distributed over the entire X-ray/[O IV] range. This provides evidence that high nuclear obscuration plays a crucial role in determining the probability of maser detection. Furthermore, after normalization with [O IV], we find a similar but weaker trend for the distribution of the maser detection rate with the absorption of the $7 \mu \mathrm{m}$ dust continuum. This suggests that the obscuration of the $7 \mu \mathrm{m}$ continuum occurs on larger spatial scales than that of the X-rays. Hence, in the AGN unified model, at moderate inclinations away from edge-on, the $7 \mu \mathrm{m}$ dust absorption may occur without any proportionate X-ray absorption. The absorption of [O III] appears unrelated to maser detections. The failure to detect masers in obscured AGN is most likely due to insufficient observational sensitivity.
\end{abstract}

Key words. galaxies: Seyfert - galaxies: nuclei - masers - X-rays: galaxies - infrared: galaxies

\section{Introduction}

$\mathrm{H}_{2} \mathrm{O}$ megamaser galaxies represent an extreme subclass of active galactic nuclei (AGN) with strong water maser emission at $22 \mathrm{GHz}$ (reviews by Lo 2005; and Henkel et al. 2005). In those cases where the emission arises from a molecular disk and can be resolved spatially using very long baseline interferometry, the central black hole $(\mathrm{BH})$ mass, and the distance to the galaxy can be determined (e.g. for NGC 4258, Greenhill et al. 1993; Herrnstein et al. 1999). Thus, finding megamasers (henceforth simply called masers) and understanding their properties is of great interest.

Owing to theoretical considerations, a large line-of-sight column density of velocity coherent gas favors the detection of a maser. High velocity coherence of the maser emitting gas is required, because energy and momentum conservation imply that the induced photon has the same frequency and direction as the stimulating photon (e.g. Elitzur 2002). While the emission of an individual maser spot is directional (i.e. beamed), a collection of these spots statistically may be expected to radiate in all directions, but this has not yet been confirmed. The originally discovered water maser emission from AGN comes from (presumably edge-on) disks, and the resolved emission in most sources traces accretion disks and in a few cases star formation masers. However, two sources, Circinus and NGC 3079, exhibit in addition off-disk jet masers that seem to trace outflows. These

* Full Table 1 is only available in electronic form at the CDS via anonymous ftp to cdsarc.u-strasbg.fr (130.79.128.5) or via http: //cdsarc.u-strasbg.fr/viz-bin/qcat?J/A+A/530/A147 outflow masers are potentially torus clouds (Nenkova et al. 2008).

In the AGN unified model, an optically thick obscuring dust torus is envisioned to encircle the accretion disk and type-1 AGN are seen pole-on while type-2 AGN are seen edge-on (Antonucci 1993). Masers are almost exclusively found in AGN of Seyfert-2 or LINER type, consistent with the picture that masers are preferentially beamed in the plane of the torus (Braatz et al. 1997, 2004; Henkel et al. 2005). But not all type-2 AGN are masers.

We note that the $22 \mathrm{GHz}$ radio-frequency maser emission itself is believed to be largely unaffected by absorption; but high $\mathrm{X}$-ray, optical, or mid-infrared obscuration may indicate that there is a high likelihood that the masing disk is seen edge-on, hence favoring a maser detection.

Type-2 AGN that host masers show a prevalence $(>80 \%)$ of high X-ray obscuring columns $\left(N_{\mathrm{H}}>10^{23} \mathrm{~cm}^{2}\right)$ and about half are Compton thick $\left(N_{\mathrm{H}}>10^{24} \mathrm{~cm}^{2}\right)$ (Braatz et al. 1997; Zhang et al. 2006; Greenhill et al. 2008). However, as pointed out by Zhang et al. (2006), among type-2 AGN the average X-ray derived column densities of masers and non-masers ${ }^{1}$ are indistinguishable. One explanation of this unexpected result could be that X-ray scattering in clumpy media dilutes the true line-ofsight column density, and thus prevents us from deriving unbiased orientation information. Therefore, it is vital to include information from other than X-ray wavelengths to reveal the potential influence of nuclear obscuration on the maser detection and non-detection, respectively.

\footnotetext{
1 We denote as non-masers those AGN that have been observed at $22 \mathrm{GHz}$, but for which no megamaser was detected.
} 
Zhang et al. (2010) analyzed the $\mathrm{K} \alpha$ iron-line equivalent width $E W(\mathrm{~K} \alpha)$, following the strategy of Bassani et al. (1999), and compared it with two optical thickness parameters, the infrared 6-400 $\mu \mathrm{m}$ luminosity $L_{\mathrm{IR}}$ derived from IRAS $12-100 \mu \mathrm{m}$ photometry and the [O III] $5007 \AA$ emission-line luminosity $L_{[\mathrm{O} \text { III] }}$. Both parameters were adopted as isotropic tracers of the intrinsic AGN strength. While the $E W(\mathrm{~K} \alpha)$ distributions of 19 masers and 34 non-masers cover the same broad range (100$3000 \mathrm{eV})$, the median $E W(\mathrm{~K} \alpha)$ of masers is about a factor of four higher than that of the non-masers, indicating that the X-ray continuum of masers is more absorbed than that of non-masers.

However, it remains a matter of debate whether $L_{\mathrm{IR}}$ and $L_{[\mathrm{O} \text { III }]}$ are indeed isotropic tracers of the intrinsic AGN luminosity. While [O III] has often been used as an isotropic AGN tracer (Mulchaey et al. 1994; Alonso-Herrero et al. 1997; Bassani et al. 1999; Heckman et al. 2005; Panessa et al. 2006; Lamastra et al. 2009), the discovery of polarized [O III] emission in some type-2 AGN (di Serego Alighieri et al. 1997) implies that a substantial fraction of the [O III] emission might be shielded by the torus. Further studies, using MIR emission lines such as [O IV] or $[\mathrm{Ne} \mathrm{V}]$ as orientation independent tracers of the AGN power, provide evidence in terms of the $[\mathrm{O} \mathrm{III}] /[\mathrm{OIV}]$ ratio, that $[\mathrm{O}$ III] suffers orientation-dependent extinction, up to a factor of ten in individual cases (Haas et al. 2005; Meléndez et al. 2008a; Baum et al. 2010).

This is qualitatively consistent with results obtained using the (extinction-corrected) $2-10 \mathrm{keV} \mathrm{X-ray} \mathrm{luminosity} L_{\mathrm{X}}$ as an intrinsic measure of AGN power; Netzer et al. (2006) find that $L_{[\mathrm{O} \mathrm{III]}} / L_{\mathrm{X}}$ of type-2 AGN is, on average, about a factor of two lower than that of type-1 AGN. Even in the face-on Sy1 case, the back-sided cone of the NLR lies - at least partly - behind an absorbing layer (e.g. the dust torus). Therefore, it is highly questionable how far [O III] can serve as an isotropic AGN tracer. The extinction correction via Balmer decrement $\left(\mathrm{H}_{\alpha} / \mathrm{H}_{\beta}=3\right)$ remains highly uncertain, since it is dependent on the geometry of the emitting and obscuring regions.

Hes et al. (1996) and Baker (1997) caution against the use of [O III] as a measure of the intrinsic NLR emission and suggest using [OII] 3727 instead. Observations of radio-loud AGN, where the orientation can be inferred from radio morphology, show that [O II] is largely orientation independent (Hes et al. 1996; Baker 1997). On the other hand, because of its low ionization potential, [O II] may also be dominated by star formation in the host (e.g. Ho 2005). Then, the decline in $L_{[\mathrm{OII}]} / L_{\mathrm{X}}$ with increasing $L_{X}$, as found by Netzer et al. (2006), could be naturally explained by a decline in host/AGN with increasing AGN $L_{X}$.

Likewise the mid-infrared $(\lambda<40 \mu \mathrm{m})$ part of $L_{\mathrm{IR}}$ is orientation dependent (e.g. Fig. 16 in Buchanan et al. 2006), while the far-infrared $(\lambda>40 \mu \mathrm{m})$ emission of Seyfert galaxies and low-luminosity quasars is dominated by star-forming contributions rather than the AGN itself (e.g. Maiolino \& Rieke 1995; Schweitzer et al. 2006). Thus, a careful re-investigation using more suitable isotropic AGN tracers would be desirable.

Here, we revisit the connection between maser detection rate and nuclear obscuration by using the strength of the [O IV] $25.9 \mu \mathrm{m}$ emission line (for short [O IV]) as tracer for the intrinsic AGN strength. [O IV] has been found to be largely unaffected by obscuration (e.g., Genzel et al. 1998; Haas et al. 2005; Meléndez et al. 2008a; Baum et al. 2010). We combine the strategies of Bassani et al. (1999) and Meléndez et al. (2008a). The observed X-ray (2-10 keV) flux normalized by [O IV] should provide information about X-ray absorption, even in the case of X-ray scattering caused by a complex geometry or for Compton thick cases. We compare the distribution of
$\mathrm{X}$-ray/[O IV] for masers and non-masers. In addition, after normalization of the $7 \mu \mathrm{m}$ flux by [O IV], we inspect the relation between maser detection rate and the absorption of the $7 \mu \mathrm{m}$ dust continuum emitted from the nuclear torus, as well as maser detection and the absorption of the [O III] $5007 \AA$ emission from the central part of the narrow-line region (NLR).

The distances from which we derived the luminosities are taken from the NED database. The cosmology is based on $H_{0}=$ $73 \mathrm{~km} \mathrm{~s}^{-1} \mathrm{Mpc}^{-1}, \Omega_{\Lambda}=0.73$, and $\Omega_{\mathrm{m}}=0.27$.

\section{Data}

\subsection{The parent sample}

At first glance, one could take all known masers and nonmasers from the literature and compare their properties, such as $L_{\mathrm{X}} / L_{\text {[O IV] }}$. However, to determine nuclear obscuration, one also needs to know the range of $L_{\mathrm{X}} / L_{[\mathrm{OIV}]}$ for unobscured (preferentially Sy1) sources, which should comprise a complete sample free of any selection bias. However, the selection of Seyferts for which a maser search has been performed has not followed any clear criteria. Even worse, most maser searches have been performed on Sy2s, but only a small number on Sy1s. Because incomplete sample selection may influence the results, we decided to start with complete Seyfert catalogs with well-defined selection criteria. To increase the sample size, we created a master sample from the following three catalogs, consisting of a total of 163 sources:

- the magnitude-limited complete sample of the CfA Redshift Survey by Huchra \& Burg (1992), which was supplied with updated Seyfert-type information from the NED database;

- the $12 \mu \mathrm{m}$ active galaxy sample by Spinoglio \& Malkan (1989), complemented by that of Rush \& Malkan (1993);

- the IRAS F25/F60 flux-ratio selected sample by de Grijp et al. (1992), as refined by Schmitt et al. (2003).

Table 1 documents how the 163 sources are distributed over the three catalogs, and how these catalogs match or complement each other. In general, we present the results for the combined sample, but - where necessary - also for the catalogs individually (Table 2).

In Sect. 3.4, we discuss potential differences between the three samples and our combined sample and all other known masers outside it. The Spitzer data archive contains IRS spectra (at $\sim 26 \mu \mathrm{m}$ ) for 126 of the 163 sources classified as Seyferts according to the NED. This data is listed in Table 3. It covers the complete CfA-sample of 54 Seyfert Galaxies. It includes 107 of 118 Seyferts (two blazars included as Sy1) from the $12 \mu \mathrm{m}$ selected sample. For the IRAS sample, we found useful IRS spectra for 34 of 60 sources.

\subsection{Maser information}

The parent sample of 126 sources with Spitzer spectra was searched for known maser-detections and non-detections. For this purpose, we used the lists compiled by Bennert et al. (2009) and those on the website of the Hubble constant Maser Experiment (HoME) ${ }^{2}$.

\footnotetext{
2 http://www. cfa.harvard.edu/ lincoln/demo/HoME/ surveys/survey.html compiled from Kondratko et al. (2006b,a);
} 
M. Ramolla et al.: Megamaser detection and nuclear obscuration in Seyfert galaxies

Table 1. Documentation of how far the three samples (of 163 sources in total) match and complement each other.

\begin{tabular}{lcccccc}
\hline \hline \multicolumn{1}{c}{$(1)$} & $(2)$ & $(3)$ & $(4)$ & $(5)$ & $(6)$ & $(7)$ \\
Intersecting sample & CfA & $12 \mu \mathrm{m}$ & IRAS & $12 \mu \mathrm{m} \cup$ IRAS & CfA U IRAS & CfA U $12 \mu \mathrm{m}$ \\
\hline CfA & 54 & 42 & 12 & 45 & - & - \\
$12 \mu \mathrm{m}$ & - & 118 & 24 & - & 57 & - \\
IRAS F25/F60 & - & - & 60 & - & - & 27 \\
\hline
\end{tabular}

Notes. Full table available at the CDS. For each row, the table entries list the number of sources contained in the intersection of the sample denoted by Cols. 2-7 with the CfA, $12 \mu \mathrm{m}$, and IRAS F25/F60 sample (Col. 1). For instance the union of the $12 \mu \mathrm{m}$ and IRAS F25/F60 samples (Col. 5) has 45 sources in common with the CfA sample (Row 1).

Table 2. The average values and standard deviations of the logarithmic luminosity ratios for each subset of Seyfert galaxies.

\begin{tabular}{|c|c|c|c|c|c|c|c|c|}
\hline \multirow{2}{*}{\multicolumn{2}{|c|}{ 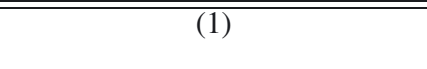 }} & \multirow{2}{*}{\multicolumn{2}{|c|}{$\begin{array}{c}(2) \\
L_{\mathrm{X}} / L_{\text {[O IV }]}\end{array}$}} & \multirow{2}{*}{\multicolumn{2}{|c|}{$\begin{array}{c}(3) \\
L_{7 \mu \mathrm{m}} / L_{[\mathrm{O} \text { IV }]}\end{array}$}} & \multirow{2}{*}{\multicolumn{2}{|c|}{$\begin{array}{c}(4) \\
L_{[\mathrm{O} \text { III }]} / L_{[\mathrm{OIV}]}\end{array}$}} & \multirow{3}{*}{$\begin{array}{c}(5) \\
\text { Parent Sample } \\
\text { Number }\end{array}$} \\
\hline & & & & & & & & \\
\hline Parent Sample & Subset & Number & $\log$ (Ratio) & Number & $\log$ (Ratio) & Number & $\log$ (Ratio) & \\
\hline \multirow[t]{6}{*}{ CfA } & Sy1 maser & 3 & $0.8 \pm 0.9$ & 3 & $1.6 \pm 0.1$ & 3 & $0.0 \pm 0.6$ & 3 \\
\hline & Sy1 non-maser & 16 & $2.0 \pm 0.5$ & 14 & $1.9 \pm 0.5$ & 16 & $0.1 \pm 0.4$ & 18 \\
\hline & Sy1 unknown & 2 & $1.73 \pm 0.02$ & 3 & $2.1 \pm 0.6$ & 2 & $-0.1 \pm 0.1$ & 4 \\
\hline & Sy2 maser & 4 & $0.1 \pm 0.5$ & 4 & $1.2 \pm 0.7$ & 4 & $-0.2 \pm 0.6$ & 4 \\
\hline & Sy2 non-maser & 16 & $1.1 \pm 0.9$ & 18 & $1.3 \pm 0.5$ & 20 & $-0.2 \pm 0.5$ & 24 \\
\hline & Sy2 unknown & 0 & - & 1 & 1.4 & 1 & -1.3 & 1 \\
\hline \multirow[t]{6}{*}{$12 \mu \mathrm{m}$} & Sy1 maser & 2 & $1.25 \pm 0.93$ & 2 & $1.6 \pm 0.1$ & 2 & $0.4 \pm 0.5$ & 2 \\
\hline & Sy1 non-maser & 22 & $2.0 \pm 0.6$ & 22 & $1.8 \pm 0.5$ & 27 & $0.1 \pm 0.4$ & 30 \\
\hline & Sy1 unknown & 4 & $2.3 \pm 0.6$ & 5 & $1.9 \pm 0.6$ & 6 & $0.0 \pm 0.3$ & 9 \\
\hline & Sy2 maser & 11 & $0.5 \pm 0.7$ & 10 & $1.2 \pm 0.6$ & 11 & $-0.3 \pm 0.5$ & 13 \\
\hline & Sy2 non-maser & 29 & $0.9 \pm 1.0$ & 34 & $1.4 \pm 0.5$ & 42 & $-0.4 \pm 0.6$ & 51 \\
\hline & Sy2 unknown & 1 & 1.1 & 1 & 1.4 & 2 & $-0.7 \pm 0.9$ & 2 \\
\hline \multirow[t]{6}{*}{ IRAS } & Sy1 maser & 0 & - & 0 & - & 0 & $\overline{-}$ & 0 \\
\hline & Sy1 non-maser & 13 & $2.2 \pm 0.4$ & 10 & $1.7 \pm 0.3$ & 15 & $0.0 \pm 0.4$ & 15 \\
\hline & Sy1 unknown & 0 & - & 0 & - & 0 & - & 0 \\
\hline & Sy2 maser & 8 & $0.2 \pm 1.0$ & 8 & $1.2 \pm 0.7$ & 8 & $-0.3 \pm 0.4$ & 8 \\
\hline & Sy2 non-maser & 6 & $0.7 \pm 0.8$ & 9 & $1.3 \pm 0.5$ & 10 & $-0.2 \pm 0.5$ & 11 \\
\hline & Sy2 unknown & 0 & - & 0 & - & 0 & - & 0 \\
\hline Combined: & Sy1 maser & 3 & $.8 \pm 0.9$ & 3 & $1.6 \pm 0.1$ & 3 & $0.0 \pm 0.6$ & 3 \\
\hline CfA U & Sy1 non-maser & 28 & $2.0 \pm 0.6$ & 25 & $1.8 \pm 0.5$ & 33 & $0.1 \pm 0.4$ & 36 \\
\hline $12 \mu \mathrm{m} \cup$ & Sy1 unknown & 5 & $2.1 \pm 0.6$ & 6 & $1.9 \pm 0.5$ & 7 & $0.0 \pm 0.3$ & 10 \\
\hline \multirow[t]{3}{*}{ IRAS } & Sy2 maser & 13 & $0.4 \pm 0.8$ & 12 & $1.1 \pm 0.6$ & 13 & $-0.4 \pm 0.5$ & 15 \\
\hline & Sy2 non-maser & 32 & $0.9 \pm 1.0$ & 42 & $1.4 \pm 0.5$ & 50 & $-0.3 \pm 0.6$ & 60 \\
\hline & Sy2 unknown & 1 & 1.1 & 1 & 1.4 & 2 & $-0.7 \pm 0.9$ & 2 \\
\hline
\end{tabular}

Notes. Column 1: in descending order, the optically selected CfA sources (Huchra \& Burg 1992), the MIR-selected $12 \mu \mathrm{m}$ sources (Rush \& Malkan 1993), the IRAS F25/F60 flux-ratio selected sources (Schmitt et al. 2003) and the combined sample that is used in this work. Columns $2-$ 4: number of sources with luminosities available in [O IV] and one of the following: 2-10 keV X-rays (Col. 2) or $7 \mu \mathrm{m}(\mathrm{Col} .3)$ or [O III] (Col. 4). Each sample's row is subdivided into Sy1 maser, non-maser, maser-unknown and Sy2 maser, non-maser, and maser-unknown. Column 5: total number of objects in the parent sample from Col. 1, e.g. 3 Sy1 maser in the CfA, 18 Sy1 non-maser in the CfA, and so forth.

This search results in 18 masers ( 3 Sy1s, 15 Sy2s), 96 nonmasers (36 Sy1s, 60 Sy2s), and 12 sources (10 Sy1s, 2 Sy2s) for which no maser search has yet been performed (henceforth called maser-unknown). The results are listed in Table 3, Col. 2.

\section{3. $[O I V] 25.89 \mu m$ line and $7 \mu m$ continuum flux}

Our analysis is based on public archival IRS spectra of Seyfert galaxies. We used the post-basic-calibration data (PBCD), reduced by the Spitzer Science Center's (SSC) pipeline. This

Hagiwara et al. (2003); Zhang et al. (2006); Braatz et al. (2004); Hagiwara et al. (2002); Greenhill et al. (2002, 1997); Sato et al. (2005); Braatz et al. (1996); Greenhill et al. (1995); Nakai et al. (1995); Henkel et al. (1998, 2005); Braatz et al. (2003); Henkel et al. (1984); Haschick \& Baan (1985); Claussen \& Lo (1986); Henkel et al. (1986); Becker et al. (1993); Greenhill et al. (1990); Braatz \& Gugliucci (2008); Henkel (2008); Braatz (2008). included droop-, stray-light-, cross-talk- and saturation correction, dark subtraction, flatfielding and coaddition.

Where possible, IRS high resolution spectra with $R \sim 600$ were chosen, to avoid the contamination of $[\mathrm{OIV}]_{25.89 \mu \mathrm{m}}$ by the neighboring $[\mathrm{Fe} \mathrm{II}]_{25.99 \mu \mathrm{m}}$ emission line. If high resolution spectra were not available, the low-resolution spectra were used, after a background subtraction had been performed by the SSC pipeline.

For the high resolution data collected with the shorter $(4.7 \times$ 11.3 and $11.1 \times 22.3$ arcsec) slits, separate background observations had to be chosen to evaluate the background contribution. This was performed in Ramolla (2009) by comparing the background with the source fluxes at the presumably weakest part of the source spectrum in the 9 and $10 \mu \mathrm{m}$ rest frame; with the result that the background contribution is negligible compared to the conservatively assumed flux calibration errors of $15 \%$. The resulting errors are calculated from an assumed $15 \%$ flux calibration error and the error in the line-fitting routine. 
The [OIV] flux was extracted by fitting a simple spectral model to a wavelength window of $\sim 0.3 \mu \mathrm{m}$ around the [O IV] line. This model consists of a linear base, convolved with Gaussian profiles that also include the neighboring [Fe II] line. No [O IV] aperture corrections had to be applied, because for both, high- and low-resolution data, the slit apertures cover an area larger than the expected size of the NLR, as estimated from the relationship ${ }^{3}$ of [O III] luminosity to NLR size, found by Bennert et al. (2002).

We calculated the $7.6 \mu \mathrm{m}$ (henceforth for short $7 \mu \mathrm{m}$ ) continuum flux from the background-subtracted IRS low-resolution spectra. We used a modified version of the PAHFIT code by Smith et al. (2007), which estimated the continuum in the 5 to $11.8 \mu \mathrm{m}$ branch. As suggested by Smith \& Draine (2008), we did not correct the continuum fit for the silicate feature at $9.7 \mu \mathrm{m}$. The $7 \mu$ m continuum flux was then calculated from the PAH flux and the equivalent width of the features at $7.4 \mu \mathrm{m}, 7.6 \mu \mathrm{m}$, and $7.8 \mu \mathrm{m}$ (Ramolla 2009). The uncertainties in the $7 \mu \mathrm{m}$ continuum are conservatively estimated to be smaller than $30 \%$, which is sufficient for our purpose. In a few cases, the AGN contribution may be contaminated by nuclear $\left(<3.7^{\prime \prime}\right)$ star formation (e.g. Deo et al. 2009). We verified that the effect on our statistical analysis is negligible compared to high resolution ground-based MIR observations.

The [OIV] and $7 \mu \mathrm{m}$ fluxes are listed in Table 3 . The values are consistent with those derived by others (Buchanan et al. 2006; Deo et al. 2007, 2009; Tommasin et al. 2010).

\subsection{X-rays $+[O I I I] 5007 \AA$ line from the literature}

The $2-10 \mathrm{keV}$ hard X-ray data were obtained by several observers using ASCA, Beppo SAX, Chandra, and XMM. We collected the data from the NED; in case of multiple entries we chose the latest detection.

We collected [O III] $5007 \AA$ emission line fluxes from various literature sources, as listed in Table 3. Because of the large uncertainties, we applied neither an extinction correction nor aperture correction for the [O III] fluxes. Those aperture corrections would affect few very nearby sources, but most sources are sufficiently distant to ensure that any bias in the statistical analysis is small.

\subsection{Additional maser sources}

On the one hand, our combined sample is drawn from the CfA, $12 \mu \mathrm{m}$, and IRAS F25/F60 catalogs, which contains 15 Sy2 maser sources with Spitzer spectra. On the other hand, a total of 52 masing Sy2 are known so far (Bennert et al. 2009; Zhang et al. 2010), although these are drawn from different AGN catalogs using inhomogeneous criteria.

To compare the 15 Sy 2 masers of our combined sample with the remaining 37, we also analyzed available Spitzer spectra and gathered additional [O III] and X-ray fluxes for them from the literature in the same manner, as we did for our combined sample. This results in an "off-sample" list of 37 Sy 2 masers that is appended to Table 3 .

\section{Results and discussion}

While our combined sample of 126 sources contains 114 maser and non-maser sources (12 maser-unknown excluded), not all of

\footnotetext{
$\overline{{ }^{3} \log \left(R_{\mathrm{NLR}}\right)=(0.52 \pm 0.06)} \times \log \left(L_{[\mathrm{O} \mathrm{III}]}\right)-(18.5 \pm 2.6)$.
}

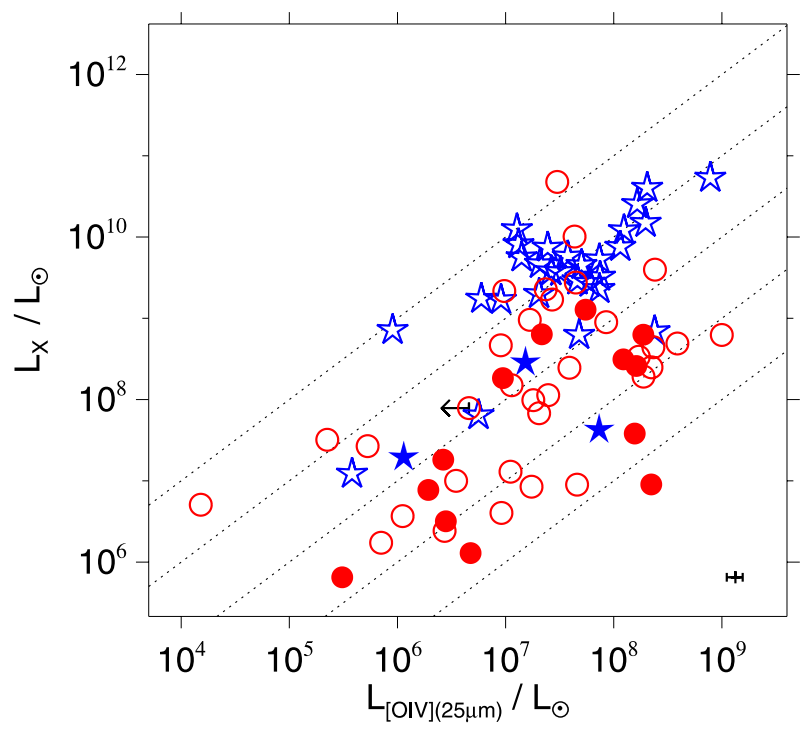

Fig. 1. Observed 2-10 keV X-ray versus [O IV] line luminosity. Blue stars represent Sy1s (Sy 1.0-1.5), red circles Sy2s (Sy 1.8-2.0). Filled symbols are masers, open symbols are non-masers. The dotted lines mark fixed $L_{\mathrm{X}} / L_{[\mathrm{O} \text { IV] }}$ ratios of $1000 ; 100 ; 10 ; 1 ; 0.1$ (from top to bottom). The error-bar in the lower right corner is the average relative error of all [OIV] measurements.

them have data for all the observables considered here (X-rays, $F_{7 \mu \mathrm{m}}$, [O III]). Therefore, we compare the maximum possible subsets of observable pairs, and discuss the implications in the framework of the AGN unified model. Therein we consider as components the accretion disk supposed to host the maser, the dust torus, the bi-conical NLR, and the host galaxy. We denote by Sy1s the subtypes between Seyfert 1.0 and 1.5, and by Sy2s those between Seyfert 1.8 and 2.0. All figures contain a combined error bar in the lower right corner that is averaged from all relative errors in this measurement. Since the literature sources do not have uniformly presented errors, we do not perform this step for the X-ray, [O III], and $\mathrm{H}_{2} \mathrm{O}$ luminosities.

\subsection{Nuclear X-ray obscuration}

Figure 1 shows the observed hard X-ray versus [O IV] line luminosities, and Fig. 2 the $L_{\mathrm{X}} / L_{[\mathrm{O} I V]}$ histogram. The main characteristics are:

- on average, Sy1s have an X-ray/[OIV] ratio that is about 10 times higher than Sy2s;

- Sy2 non-masers are evenly distributed over the entire range occupied by Sy2 masers and Sy1s (Fig. 1);

- Sy2 masers are almost disjoint from Sy1s. At a given [O IV] luminosity, Sy2 masers have on average about a factor of four lower X-ray luminosity than Sy2 non-masers (Fig. 2). Likewise, the few Sy1 masers have a lower X-ray luminosity than Sy1 non-masers ${ }^{4}$;

- six out of 12 sources without masing information, but $L_{X}$ and $L_{[O I V]}$ available (see Table 3 ), show the same trends as the Sy1s and Sy2s with masing information (see Table 2). They are not plotted, to avoid the overcrowding of Figs. 1 and 2 , with too many different symbols.

4 The Sy1 masers are NGC 4051, NGC 4151, and UCG 5101. Note that both NGC 4051 and NGC 4151 have relatively low maser $\mathrm{H}_{2} \mathrm{O}$ luminosity, and UCG 5101 is an ultra-luminous infrared galaxy so that the maser luminosity could arise from starburst regions rather than from the AGN accretion disk. 
M. Ramolla et al.: Megamaser detection and nuclear obscuration in Seyfert galaxies

Table 3. Measured fluxes and literature values.

\begin{tabular}{|c|c|c|c|c|c|c|}
\hline (1) & (2) & (3) & (4) & (5) & (6) & (7) \\
\hline \multirow[t]{2}{*}{ Source } & $\mathrm{H}_{2} \mathrm{O}$ & Sy & [O IV] & [O III] & $2-10 \mathrm{keV}$ & $7.6 \mu \mathrm{m}$ \\
\hline & $\log \left(\frac{L}{L_{\odot}}\right)$ & & $\left(10^{-15} \mathrm{~s}\right.$ & $\left.\frac{\mathrm{erg}}{\mathrm{scm}^{2}}\right)$ & $\left(10^{-14}\right.$ & $\left.4 \frac{\mathrm{erg}}{\mathrm{scm}^{2}}\right)$ \\
\hline $\operatorname{MRK} 334^{[a]}$ & - & 1.8 & $82 \pm 14$ & $49^{[1]}$ & $800^{[2]}$ & $504 \pm 101$ \\
\hline MRK $335^{[a, b]}$ & - & 1.0 & $67 \pm 10$ & $950^{[3]}$ & $960^{[4]}$ & $983 \pm 241$ \\
\hline MRK $938^{[b]}$ & * & 2.0 & - & $44^{[5]}$ & $23^{[6]}$ & $332 \pm 66$ \\
\hline $\mathrm{E} 12-\mathrm{G} 21^{[b]}$ & $?$ & 1.0 & $187 \pm 56$ & $97^{[7]}$ & - & $366 \pm 82$ \\
\hline $\operatorname{MRK} 348^{[b, c]}$ & 2.6 & 2.0 & $163 \pm 25$ & $359^{[8]}$ & $482^{[9]}$ & $1151 \pm 235$ \\
\hline $\operatorname{IZw} 1^{[b]}$ & $?$ & 1.0 & $97 \pm 13$ & $44^{[1]}$ & $680^{[10]}$ & - \\
\hline IRAS $00521-7054^{[b]}$ & - & 2.0 & $71 \pm 11$ & $77^{[8]}$ & - & - \\
\hline $\mathrm{NGC} 424^{[b]}$ & - & 2.0 & $223 \pm 34$ & $420^{[3]}$ & $122^{[11]}$ & $3655 \pm 738$ \\
\hline $\mathrm{NGC} 526 \mathrm{~A}^{[b]}$ & - & 1.5 & $176 \pm 26$ & $270^{[3]}$ & $2046^{[11]}$ & $669 \pm 162$ \\
\hline $\operatorname{NGC} 513^{[b]}$ & - & 2.0 & $59 \pm 9$ & $35^{[12]}$ & - & $232 \pm 59$ \\
\hline F01475-0740 $0^{[b, c]}$ & - & 2.0 & $62 \pm 10$ & $53^{[8]}$ & $82^{[13]}$ & $393 \pm 111$ \\
\hline $\mathrm{UM} 146^{[a]}$ & - & 1.9 & $26 \pm 3$ & $60^{[3]}$ & - & $<199$ \\
\hline MRK $590^{[c]}$ & - & 1.2 & $31 \pm 8$ & $53^{[1]}$ & $1970^{[14]}$ & $<589$ \\
\hline MCG $+05-06-036^{[a, b]}$ & $?$ & 1.0 & $42 \pm 5$ & - & - & $166 \pm 33$ \\
\hline $\mathrm{NGC} 931^{[b, c]}$ & - & 1.5 & $459 \pm 67$ & $75^{[8]}$ & $2000^{[15]}$ & $1697 \pm 392$ \\
\hline $\mathrm{NGC} 1068^{[a, b, c]}$ & 2.2 & 2.0 & $18908 \pm 2697$ & $4834^{[8]}$ & $462^{[16]}$ & $52585 \pm 10567$ \\
\hline NGC $1056^{[b]}$ & - & 2.0 & $<212$ & $23^{[7]}$ & - & $235 \pm 47$ \\
\hline NGC $1097^{[a, b]}$ & - & 1.0 & $52 \pm 12$ & $18^{[7]}$ & $170^{[17]}$ & $283 \pm 58$ \\
\hline $\mathrm{NGC} 1125^{[b]}$ & - & 2.0 & $356 \pm 52$ & $23^{[18]}$ & - & $118 \pm 27$ \\
\hline $\mathrm{NGC} 1144^{[b]}$ & - & 2.0 & $69 \pm 10$ & - & $11000^{[2]}$ & $164 \pm 37$ \\
\hline M-2-8-39 $9^{[b, c]}$ & - & 2.0 & $144 \pm 21$ & $183^{[8]}$ & - & $270 \pm 69$ \\
\hline $\mathrm{NGC} 1194^{[b, c]}$ & - & 1.0 & $144 \pm 21$ & $396^{[19]}$ & - & $521 \pm 105$ \\
\hline $\mathrm{NGC} 1241^{[b]}$ & - & 2.0 & $<100$ & $370^{[\mathbf{2 0}]}$ & - & - \\
\hline $\mathrm{NGC} 1320^{[b, c]}$ & * & 2.0 & $254 \pm 37$ & $122^{[8]}$ & $496^{[21]}$ & $933 \pm 231$ \\
\hline NGC $1365^{[b]}$ & - & 1.8 & $1441 \pm 207$ & $62^{[22]}$ & $660^{[23]}$ & $2759 \pm 553$ \\
\hline $\mathrm{NGC} 1386^{[b, c]}$ & 2.1 & 2.0 & $991 \pm 145$ & $800^{[24]}$ & $27^{[18]}$ & $1017 \pm 206$ \\
\hline IRAS $03362-1641^{[b]}$ & - & 2.0 & $52 \pm 8$ & $18^{[8]}$ & - & - \\
\hline $\mathrm{F} 03450+0055^{[b]}$ & $?$ & 1.5 & $31 \pm 5$ & $100^{[25]}$ & - & $<10504$ \\
\hline $3 \mathrm{C} 120^{[a, b]}$ & - & 1.0 & $1195 \pm 174$ & $304^{[8]}$ & $8200^{[26]}$ & $987 \pm 235$ \\
\hline MRK $618^{[b]}$ & - & 1.0 & $96 \pm 16$ & $160^{[8]}$ & $700^{[27]}$ & - \\
\hline $\mathrm{F} 04385-0828^{[b]}$ & - & 2.0 & $80 \pm 14$ & $3^{[7]}$ & $1800^{[2]}$ & $1119 \pm 228$ \\
\hline NGC $1667^{[b]}$ & - & 2.0 & $68 \pm 11$ & $64^{[28]}$ & $3^{[29]}$ & $76 \pm 18$ \\
\hline $\mathrm{E} 33-\mathrm{G} 2^{[b, c]}$ & - & 2.0 & $137 \pm 20$ & $57^{[19]}$ & - & - \\
\hline M-5-13-17 $7^{[b, c]}$ & - & 1.5 & $98 \pm 15$ & $340^{[19]}$ & - & $376 \pm 96$ \\
\hline IRAS $05189-2524^{[b]}$ & - & 2.0 & $218 \pm 16$ & $39^{[\mathbf{3 0}]}$ & $360^{[31]}$ & $2247 \pm 451$ \\
\hline Markarian3 ${ }^{[c]}$ & 1.0 & 2.0 & $1763 \pm 358$ & $1070^{[8]}$ & $590^{[32]}$ & $1593 \pm 349$ \\
\hline $\operatorname{MRK} 6^{[b, c]}$ & - & 1.5 & $385 \pm 56$ & $700^{[8]}$ & $1200^{[33]}$ & - \\
\hline MRK $9^{[b]}$ & - & 1.5 & $48 \pm 8$ & $109^{[3]}$ & - & $<1944$ \\
\hline $\operatorname{MRK} 79^{[b, c]}$ & - & 1.2 & $395 \pm 57$ & $370^{[3]}$ & $2600^{[15]}$ & $<3567$ \\
\hline IRAS $07598+6508^{[a, b]}$ & $?$ & 1.0 & $<168$ & - & - & - \\
\hline MRK $622^{[c]}$ & - & 2.0 & $66 \pm 8$ & $40^{[19]}$ & $22^{[13]}$ & - \\
\hline NGC $2639^{[b]}$ & 1.4 & 1.9 & $36 \pm 4$ & $14^{[34]}$ & $25^{[35]}$ & $<155$ \\
\hline IRAS $08572+3915^{[a, b]}$ & ? & 2.0 & $167 \pm 50$ & $8^{[5]}$ & - & $427 \pm 85$ \\
\hline MRK $704^{[b]}$ & - & 1.5 & $117 \pm 18$ & $85^{[1]}$ & $537^{[11]}$ & $<10595$ \\
\hline $\mathrm{NGC} 2841^{[a]}$ & - & 1.0 & $12 \pm 3$ & - & - & $161 \pm 51$ \\
\hline $\operatorname{pg} 0923+129^{[c]}$ & - & 1.2 & $74 \pm 12$ & $90^{[19]}$ & $1151^{[11]}$ & $458 \pm 96$ \\
\hline UGC $5101^{[a]}$ & 3.2 & 1.5 & $82 \pm 11$ & $21^{[5]}$ & $5^{[36]}$ & $276 \pm 55$ \\
\hline $\mathrm{NGC} 2992^{[a, b]}$ & - & 1.9 & $1300 \pm 134$ & $360^{[1]}$ & $8030^{[37]}$ & $639 \pm 130$ \\
\hline MRK $1239^{[b]}$ & - & 1.5 & $154 \pm 24$ & $467^{[8]}$ & - & $3323 \pm 672$ \\
\hline NGC $3031^{[a, b]}$ & - & 1.8 & $44 \pm 13$ & $100^{[34]}$ & $1500^{[29]}$ & - \\
\hline $3 \mathrm{C} 234^{[b]}$ & $?$ & 1.0 & $79 \pm 12$ & - & - & $407 \pm 92$ \\
\hline NGC $3079^{[a, b]}$ & 2.7 & 2.0 & $290 \pm 53$ & $945^{[5]}$ & $33^{[16]}$ & $160 \pm 32$ \\
\hline $\mathrm{NGC} 3227^{[a, b]}$ & - & 1.5 & $655 \pm 95$ & $820^{[1]}$ & $750^{[38]}$ & - \\
\hline $\mathrm{NGC} 3281^{[c]}$ & - & 2.0 & $1779 \pm 534$ & $55^{[1]}$ & - & $162 \pm 32$ \\
\hline $\mathrm{NGC} 3393^{[c]}$ & 2.6 & 2.0 & $2214 \pm 184$ & $268^{[18]}$ & $9^{[18]}$ & $199 \pm 52$ \\
\hline $\mathrm{NGC} 3511^{[b]}$ & - & 1.0 & $23 \pm 6$ & - & - & $27 \pm 5$ \\
\hline NGC $3516^{[a, c]}$ & - & 1.5 & $451 \pm 66$ & $270^{[1]}$ & $4410^{[18]}$ & $<2900$ \\
\hline $\mathrm{M}+0-29-23^{[b]}$ & - & 2.0 & $78 \pm 23$ & $5^{[7]}$ & - & $348 \pm 69$ \\
\hline $\mathrm{NGC} 3660^{[b]}$ & - & 2.0 & $25 \pm 5$ & $33^{[28]}$ & - & $<234$ \\
\hline NGC $3783^{[a, c]}$ & - & 1.0 & $378 \pm 57$ & $763^{[8]}$ & $8500^{[39]}$ & $2261 \pm 470$ \\
\hline $\operatorname{NGC} 3786^{[a]}$ & - & 1.8 & $129 \pm 19$ & $84^{[3]}$ & - & $281 \pm 56$ \\
\hline NGC $3982^{[a, b]}$ & - & 2.0 & $89 \pm 15$ & $188^{[34]}$ & $22^{[11]}$ & $49 \pm 10$ \\
\hline NGC $4051^{[a, b]}$ & 0.3 & 1.5 & $366 \pm 53$ & $390^{[\mathbf{3 4}]}$ & $627^{[16]}$ & $1704 \pm 344$ \\
\hline UGC $7064^{[a, b]}$ & - & 1.9 & $118 \pm 17$ & - & - & $269 \pm 55$ \\
\hline NGC $4151^{[a, b]}$ & -0.2 & 1.5 & $2396 \pm 342$ & $11600^{[34]}$ & $4510^{[\mathbf{1 6}]}$ & $7211 \pm 1459$ \\
\hline $\operatorname{MRK} 766^{[a, b, c]}$ & - & 1.0 & $474 \pm 69$ & $453^{[8]}$ & $3000^{[40]}$ & $1061 \pm 213$ \\
\hline NGC $4388^{[a, b, c]}$ & 1.1 & 2.0 & $2996 \pm 644$ & $564^{[8]}$ & $762^{[16]}$ & $971 \pm 199$ \\
\hline $3 \mathrm{C} 273^{[b]}$ & $?$ & 1.0 & $79 \pm 9$ & $116^{[\mathbf{4 1}]}$ & $8300^{[15]}$ & $2043 \pm 424$ \\
\hline $\mathrm{NGC} 4501^{[a, b]}$ & - & 2.0 & $33 \pm 6$ & $34^{[34]}$ & $11^{[\mathbf{1 6}]}$ & $<271$ \\
\hline $\mathrm{NGC} 4507^{[a, c]}$ & - & 2.0 & $332 \pm 51$ & $828^{[8]}$ & $2100^{[29]}$ & $1579 \pm 320$ \\
\hline
\end{tabular}

\begin{tabular}{|c|c|c|c|c|c|c|}
\hline Source & $\mathrm{H}_{2} \mathrm{O}$ & Sy & [OIV] & [O III] & $2-10 \mathrm{keV}$ & $7.6 \mu \mathrm{m}$ \\
\hline NGC 4569 $9^{[a]}$ & - & 2.0 & $42 \pm 10$ & $24^{[5]}$ & - & $463 \pm 95$ \\
\hline $\operatorname{NGC} 4579^{[a, b]}$ & - & 1.9 & $30 \pm 5$ & - & $440^{[29]}$ & $325 \pm 74$ \\
\hline $\mathrm{NGC} 4593^{[b, c]}$ & _- & 1.0 & $127 \pm 40$ & $134^{[8]}$ & $3710^{[\mathbf{4 2}]}$ & $1428 \pm 304$ \\
\hline NGC $4602^{[b]}$ & - & 1.9 & $<66$ & $134^{[8]}$ & - & $70 \pm 18$ \\
\hline TOL $1238-364^{[b]}$ & _- & 2.0 & $145 \pm 23$ & $194^{[18]}$ & $17^{[11]}$ & $779 \pm 161$ \\
\hline $\mathrm{M}-2-33-34^{[b]}$ & - & 1.0 & $670 \pm 145$ & $364^{[8]}$ & - & $175 \pm 57$ \\
\hline MRK $231^{[b]}$ & - & 1.0 & $233 \pm 70$ & $230^{[1]}$ & $68^{[11]}$ & $5960 \pm 1192$ \\
\hline NGC $4826^{[a]}$ & - & 2.0 & $139 \pm 41$ & - & _- & $540 \pm 108$ \\
\hline NGC $4922^{[b]}$ & 2.3 & 2.0 & - & $64^{[5]}$ & - & $476 \pm 96$ \\
\hline $\mathrm{NGC} 4941^{[b]}$ & - & 2.0 & $285 \pm 43$ & $143^{[18]}$ & $82^{[11]}$ & $<513$ \\
\hline $\mathrm{NGC} 4968^{[b, c]}$ & - & 2.0 & $307 \pm 45$ & $177^{[8]}$ & $15^{[13]}$ & $742 \pm 156$ \\
\hline $\mathrm{NGC} 5005^{[a, b]}$ & - & 2.0 & $179 \pm 21$ & $7^{[1]}$ & - & $344 \pm 69$ \\
\hline $\operatorname{NGC} 5033^{[a, b]}$ & - & 1.8 & $109 \pm 23$ & $53^{[1]}$ & $550^{[29]}$ & $211 \pm 43$ \\
\hline MCG-03-34-064 ${ }^{[a, b]}$ & - & 1.8 & $1062 \pm 153$ & $1507^{[8]}$ & $210^{[\mathbf{4 3}]}$ & $2206 \pm 442$ \\
\hline NGC $5135^{[b]}$ & - & 2.0 & $726 \pm 147$ & $219^{[44]}$ & - & $808 \pm 161$ \\
\hline NGC $5194^{[b]}$ & -0.2 & 2.0 & $227 \pm 47$ & $120^{[34]}$ & $48^{[16]}$ & $199 \pm 46$ \\
\hline M-6-30-15 $5^{[b, c]}$ & - & 1.2 & $227 \pm 34$ & $75^{[19]}$ & $4220^{[37]}$ & $1425 \pm 313$ \\
\hline IRAS $13349+2438^{[a, b]}$ & $?$ & 1.0 & $64 \pm 9$ & $47^{[7]}$ & $360^{[45]}$ & $3194 \pm 673$ \\
\hline MRK $266^{[b]}$ & 1.5 & 2.0 & $349 \pm 77$ & $23^{[34]}$ & $56^{[23]}$ & $72 \pm 14$ \\
\hline MRK $273^{[a, b]}$ & - & 2.0 & $474 \pm 142$ & $213^{[5]}$ & $60^{[29]}$ & - \\
\hline IC $4329 \mathrm{a}^{[a, b]}$ & - & 1.2 & $1061 \pm 156$ & $340^{[\mathbf{1 2}]}$ & $16400^{[46]}$ & $4140 \pm 968$ \\
\hline $\operatorname{NGC} 5347^{[a, b, c]}$ & 1.5 & 2.0 & $54 \pm 9$ & $45^{[8]}$ & $22^{[47]}$ & $628 \pm 129$ \\
\hline $\operatorname{MRK} 463 \mathrm{E}^{[a, b]}$ & - & 2.0 & $639 \pm 96$ & $563^{[1]}$ & $40^{[29]}$ & $2295 \pm 461$ \\
\hline NGC $5506^{[b]}$ & 1.7 & 1.9 & $2492 \pm 360$ & $521^{[28]}$ & $5800^{[48]}$ & $4222 \pm 844$ \\
\hline $\operatorname{NGC} 5548^{[a, b, c]}$ & - & 1.5 & $141 \pm 24$ & $360^{[8]}$ & $4300^{[49]}$ & $726 \pm 174$ \\
\hline $\operatorname{MRK} 817^{[a, b]}$ & - & 1.5 & $73 \pm 12$ & $140^{[1]}$ & - & $950 \pm 244$ \\
\hline PG1501+106 $[a]$ & - & 1.5 & $246 \pm 36$ & $250^{[1]}$ & $1869^{[11]}$ & - \\
\hline NGC $5929^{[a, b]}$ & - & 2.0 & $<114$ & $93^{[1]}$ & $197^{[11]}$ & $32 \pm 8$ \\
\hline NGC $5953^{[b]}$ & - & 2.0 & $172 \pm 25$ & $63^{[3]}$ & - & $259 \pm 52$ \\
\hline M-2-40-4 $4^{[b]}$ & - & 2.0 & $115 \pm 19$ & $74^{[12]}$ & $2693^{[11]}$ & $1586 \pm 382$ \\
\hline F15480-0344[b] & - & 2.0 & $364 \pm 53$ & $138^{[8]}$ & $37^{[13]}$ & $<838$ \\
\hline ESO $141-G 055^{[b]}$ & $?$ & 1.0 & $107 \pm 16$ & $164^{[8]}$ & $2650^{[\mathbf{5 0}]}$ & - \\
\hline IRAS $19254-7245^{[a, b]}$ & - & 2.0 & $105 \pm 31$ & $602^{[51]}$ & $20^{[52]}$ & $<323$ \\
\hline NGC $6810^{[b]}$ & - & 2.0 & $68 \pm 13$ & $13^{[7]}$ & - & $838 \pm 168$ \\
\hline $\mathrm{NGC} 6860^{[b, c]}$ & - & 1.0 & $122 \pm 18$ & $25^{[19]}$ & $4900^{[26]}$ & $<2201$ \\
\hline NGC $6890^{[b]}$ & _- & 2.0 & $90 \pm 13$ & $72^{[18]}$ & $8^{[11]}$ & $410 \pm 97$ \\
\hline MRK $509^{[a, b]}$ & - & 1.2 & $286 \pm 44$ & $540^{[8]}$ & $5660^{[\mathbf{5 3}]}$ & $1221 \pm 254$ \\
\hline $\mathrm{UGC} 11630^{[c]}$ & _- & 2.0 & $175 \pm 28$ & _- & _- & $280 \pm 77$ \\
\hline IC $5063^{[a, b, c]}$ & - & 2.0 & $1139 \pm 167$ & $564^{[8]}$ & $1200^{[29]}$ & $2949 \pm 598$ \\
\hline UGC $11680^{[b]}$ & - & 2.0 & $45 \pm 13$ & $88^{[54]}$ & - & $<686$ \\
\hline PG 2130+099[a] & ? & 1.0 & $103 \pm 16$ & $104^{[8]}$ & $530^{[55]}$ & $861 \pm 277$ \\
\hline IC $5135^{[b]}$ & - & 2.0 & $300 \pm 40$ & $27^{[30]}$ & $6^{[36]}$ & $451 \pm 90$ \\
\hline $\mathrm{NGC} 7172^{[b]}$ & - & 2.0 & $384 \pm 39$ & $10^{[56]}$ & $2200^{[6]}$ & $522 \pm 104$ \\
\hline IRAS $22017+0319^{[b]}$ & $?$ & 2.0 & $287 \pm 42$ & $218^{[8]}$ & $360^{[57]}$ & - \\
\hline $\operatorname{NGC} 7213^{[a, b, c]}$ & - & 1.5 & $45 \pm 8$ & $130^{[8]}$ & $3660^{[\mathbf{1 4}]}$ & $798 \pm 202$ \\
\hline $3 \mathrm{C} 445^{[a, b]}$ & - & 1.0 & $71 \pm 14$ & - & $700^{[\mathbf{5 8}]}$ & $765 \pm 242$ \\
\hline NGC $7314^{[a, b]}$ & - & 1.9 & $690 \pm 101$ & $61^{[3]}$ & $3560^{[29]}$ & $249 \pm 65$ \\
\hline UGC $12138^{[a, c]}$ & - & 1.8 & $105 \pm 15$ & $144^{[8]}$ & - & $273 \pm 57$ \\
\hline M-3-58-7 $\left[{ }^{b]}\right.$ & - & 2.0 & $117 \pm 19$ & $251^{[7]}$ & - & $1186 \pm 272$ \\
\hline NGC $7469^{[a, b]}$ & - & 1.2 & $322 \pm 48$ & $840^{[1]}$ & $2900^{[49]}$ & $2298 \pm 460$ \\
\hline NGC $7582^{[a, b]}$ & - & 2.0 & $2449 \pm 587$ & $300^{[1]}$ & $1550^{[29]}$ & $309 \pm 61$ \\
\hline $\mathrm{NGC} 7590^{[b]}$ & - & 2.0 & $58 \pm 18$ & $11^{[18]}$ & - & $70 \pm 17$ \\
\hline $\mathrm{NGC} 7603^{[a, b]}$ & - & 1.5 & $24 \pm 4$ & $29^{[1]}$ & $2400^{[14]}$ & $1619 \pm 339$ \\
\hline $\mathrm{NGC} 7674^{[a, b, c]}$ & - & 2.0 & $448 \pm 110$ & $718^{[8]}$ & $50^{[29]}$ & $1095 \pm 248$ \\
\hline NGC $7679^{[a]}$ & - & 1.0 & $350 \pm 36$ & $472^{[5]}$ & $458^{[11]}$ & - \\
\hline CGCG381-051 ${ }^{[b]}$ & - & 2.0 & $<72$ & $5^{[8]}$ & - & $317 \pm 102$ \\
\hline \multicolumn{7}{|c|}{ Additional off-sample data } \\
\hline NGC 253 & -0.8 & 2.0 & $1519 \pm 239$ & - & - & - \\
\hline NGC 449 & 1.7 & 2.0 & - & $330^{[1]}$ & $13^{[13]}$ & - \\
\hline NGC 591 & 1.4 & 2.0 & - & $1780^{[3]}$ & $18^{[\mathbf{1 1}]}$ & - \\
\hline NGC 613 & 1.3 & 2.0 & - & - & - & - \\
\hline IC 184 & 1.4 & 2.0 & - & - & - & - \\
\hline NGC 1052 & 2.1 & 2.0 & - & - & $112^{[36]}$ & - \\
\hline NGC 1106 & 0.9 & 2.0 & - & - & - & - \\
\hline MRK 1066 & 1.5 & 2.0 & - & $5140^{[3]}$ & $36^{[13]}$ & - \\
\hline IRAS $03355+0104$ & 2.7 & 2.0 & - & $76^{[8]}$ & - & - \\
\hline IC 342 & -2.0 & 2.0 & - & $34^{[59]}$ & - & - \\
\hline UGC 3255 & 1.2 & 2.0 & - & - & - & - \\
\hline VIIZw73 & 2.2 & 2.0 & _- & $74^{[8]}$ & _- & _- \\
\hline NGC 2273 & 0.8 & 2.0 & $187 \pm 56$ & $330^{[3]}$ & $69^{[18]}$ & $1014 \pm 204$ \\
\hline MRK 78 & 1.5 & 2.0 & $792 \pm 82$ & $653^{[60]}$ & - & $422 \pm 91$ \\
\hline MRK 1210 & 1.9 & 2.0 & $209 \pm 26$ & $285^{[18]}$ & $840^{[\mathbf{6 1}]}$ & $1244 \pm 260$ \\
\hline 2MASXJ08362280 & 3.4 & 2.0 & - & - & - & - \\
\hline
\end{tabular}


Table 3. continued

\begin{tabular}{|c|c|c|c|c|c|c|}
\hline Source & $\mathrm{H}_{2} \mathrm{O}$ & Sy & {$[\mathrm{OIV}]$} & [O III] & $2-10 \mathrm{keV}$ & $7.6 \mu \mathrm{m}$ \\
\hline NGC 2979 & 2.1 & 2.0 & - & $11^{[18]}$ & - & - \\
\hline IC 2560 & 2.0 & 2.0 & $558 \pm 85$ & $125^{[18]}$ & $39^{[\mathbf{6 2}]}$ & $469 \pm 115$ \\
\hline MRK 34 & 3.0 & 2.0 & $626 \pm 76$ & $520^{[1]}$ & $35^{[11]}$ & $244 \pm 67$ \\
\hline NGC 3735 & 1.3 & 2.0 & - & $330^{[59]}$ & - & - \\
\hline NGC 4258 & 1.9 & 1.9 & $76 \pm 13$ & $100^{[63]}$ & $837^{[16]}$ & $576 \pm 132$ \\
\hline ESO $269-12$ & 3.0 & 2.0 & - & $6^{[18]}$ & - & - \\
\hline NGC 4945 & 1.7 & 2.0 & $320 \pm 49$ & - & $1300^{[\mathbf{6 4}]}$ & - \\
\hline NGC 5495 & 2.3 & 2.0 & - & - & - & - \\
\hline Circinus & 1.3 & 2.0 & $8599 \pm 1231$ & $83^{[\mathbf{6 5}]}$ & $1400^{[66]}$ & $39011 \pm 7803$ \\
\hline NGC 5643 & 1.4 & 2.0 & $940 \pm 142$ & $800^{[1]}$ & $84^{[18]}$ & - \\
\hline NGC 5728 & 1.9 & 1.9 & $1162 \pm 117$ & $115^{[\mathbf{1 8}]}$ & $133^{[6]}$ & $220 \pm 44$ \\
\hline NGC 5793 & 2.0 & 2.0 & - & - & $13^{[11]}$ & - \\
\hline NGC 6240 & 1.6 & 2.0 & $236 \pm 35$ & $202^{[5]}$ & $91^{[36]}$ & $483 \pm 96$ \\
\hline NGC 6264 & 3.1 & 2.0 & - & $3200^{[67]}$ & - & - \\
\hline NGC 6323 & 2.7 & 2.0 & - & - & - & - \\
\hline NGC 6300 & 0.5 & 2.0 & $304 \pm 33$ & $140^{[68]}$ & $860^{[69]}$ & $887 \pm 177$ \\
\hline Eso 103-G035 & 2.6 & 2.0 & - & $43^{[\mathbf{1 8}]}$ & $907^{[70]}$ & - \\
\hline IRAS $19370-0131$ & 2.2 & 2.0 & - & - & - & - \\
\hline NGC 6926 & 2.7 & 2.0 & $45 \pm 7$ & $241^{[5]}$ & - & $64 \pm 13$ \\
\hline AM $2158-380$ & 2.7 & 2.0 & - & - & - & - \\
\hline NGC 7479 & 1.2 & 2.0 & $<136$ & - & $112^{[71]}$ & - \\
\hline
\end{tabular}

Notes. Column 1: source names with catalog marks: ${ }^{(a)}$ CfA sample; (b) $12 \mu \mathrm{m}$ sample; ${ }^{(c)}$ IRAS F25/F60 sample. Column 2: isotropic maser luminosities obtained from Bennert et al. (2009). A question mark designates sources unobserved for masers and a dash represents a maser undetected source. Note that MRK 938 and NGC 1320 are listed as Maser in Zhang et al. (2010) but have no luminosity information available. Therefore, they are marked with asterisks. Column 3: Seyfert type obtained from the NED or the literature with references given in square brackets. Column 4: [O IV] flux determined by Ramolla (2009). Column 5: [O III] flux from the literature. Column 6: 2-10 keV X-ray flux obtained from the literature. Column 7: $7 \mu \mathrm{m}$ continuum flux determined by Ramolla (2009). An analysis of the off-sample masers has also been performed. This data is appended in the table.

References. 1: Dahari \& De Robertis (1988). 2: Polletta et al. (1996). 3: Whittle (1992). 4: Gondoin et al. (2002). 5: Moustakas \& Kennicutt (2006). 6: Shu et al. (2007). 7: Tran (2003a). 8: de Grijp et al. (1992). 9: Awaki et al. (2000). 10: Reeves \& Turner (2000). 11: Ueda et al. (2005). 12: Tran (2003b). 13: Guainazzi et al. (2005). 14: Malizia et al. (2007). 15: Turner \& Pounds (1989). 16: Cappi et al. (2006). 17: Iyomoto et al. (1996). 18: Gu et al. (2006). 19: Schmitt et al. (2003). 20: Vaceli et al. (1997). 21: Greenhill et al. (2008). 22: VéronCetty \& Véron (2006). 23: Risaliti et al. (2000). 24: Bennert et al. (2006). 25: Boroson \& Meyers (1992). 26: Verrecchia et al. (2007). 27: Rao et al. (1992). 28: Storchi-Bergmann et al. (1995). 29: Bassani et al. (1999). 30: Kim et al. (1995). 31: Severgnini et al. (2001). 32: Bianchi et al. (2005). 33: Immler et al. (2003). 34: Ho et al. (1995). 35: Terashima et al. (2002). 36: González-Martín et al. (2006). 37: Nandra et al. (2007). 38: Gondoin et al. (2003b). 39: Blustin et al. (2002). 40: Matt et al. (2000). 41: Shang et al. (2007). 42: Perola et al. (2002). 43: Miniutti et al. (2007). 44: Phillips et al. (1983). 45: Holczer et al. (2007). 46: Gondoin et al. (2001). 47: Levenson et al. (2006). 48: Bianchi et al. (2003). 49: Reynolds (1997). 50: Gondoin et al. (2003a). 51: Buchanan et al. (2006). 52: Braito et al. (2003). 53: Perola et al. (2000). 54: Kewley et al. (2001). 55: Lawson \& Turner (1997). 56: Sharples et al. (1984). 57: Ueno et al. (2000). 58: Shinozaki et al. (2006). 59: Ho et al. (1997). 60: Whittle \& Wilson (2004). 61: Zhang \& Fan (2009). 62: Tilak et al. (2008). 63: Heckman et al. (1980). 64: Done et al. (2003). 65: Oliva et al. (1994). 66: Smith \& Wilson (2001). 67: Lumsden \& Alexander (2001). 68: Lumsden et al. (2004). 69: Matsumoto et al. (2004). 70: Turner et al. (1997). 71: Panessa et al. (2006).

We assume that the X-ray deficit, i.e. the decrease in $L_{\mathrm{X}} / L_{[\mathrm{O} I V]}$, is caused by obscuration, probably in the molecular dust torus. Figures 1 and 2 then clearly demonstrate that masers are found almost exclusively in Sy2s with heavy nuclear obscuration, while non-maser Sy2s exhibit a broad range of X-ray absorption. A two-sided Kolmogorov-Smirnov (KS) test, applied to

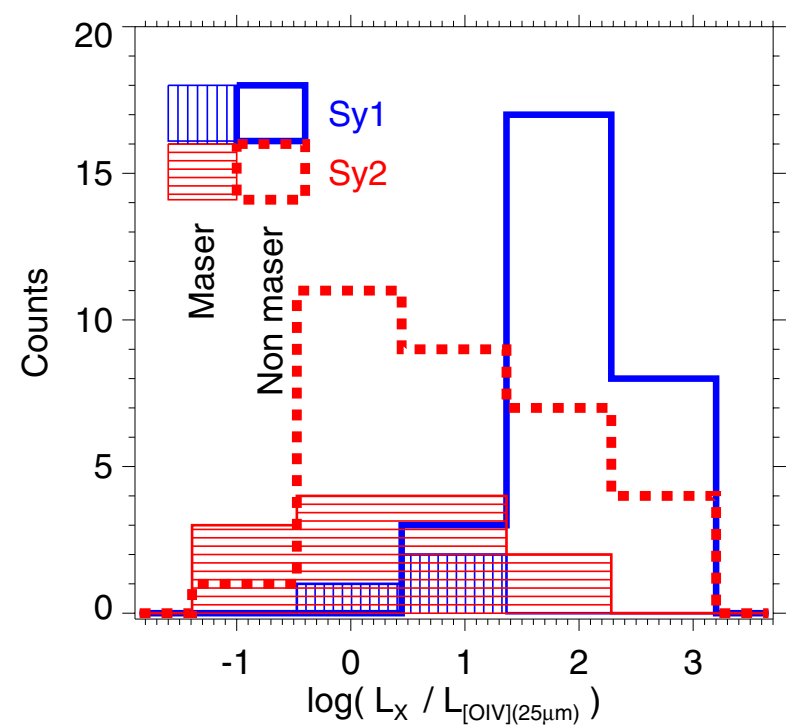

Fig. 2. Histogram of the $L_{\mathrm{X}} / L_{[\mathrm{OIV}]}$ ratio of the data points shown in Fig. 1. The red dashed line represents Sy2 non-masers, whereas the blue thick line represents the Sy1 non-masers. The maser-detections are represented by the dashed surfaces - blue and vertically dashed for Sy1, red and horizontally dashed for Sy2. The one upper limit is excluded.

the $L_{\mathrm{X}} / L_{\text {[O IV] }}$ distribution, infers a probability of $18 \%$ that the Sy 2 masers and non-masers are drawn from the same parent population. Our results agree with those of Greenhill et al. (2008) and Zhang et al. (2010), who find that about $60 \%$ of the masers are Compton-thick.

Assuming that Sy1s are almost unobscured, the obscured sources populate the $L_{\mathrm{X}} / L_{[\mathrm{OIV}]}$ range below 10 in Fig. 1 . Thus, masers almost completely populate the range of obscured sources. This range also surprisingly contains numerous nonmasers. To more clearly understand why the maser search failed in these absorbed sources, we consider the influence of observed brightness. Figure 3 shows the X-ray and [OIV] flux distribution (instead of the luminosity distribution). The Sy2 masers and non-masers exhibit a flux-dependence in their [O IV] distribution. Sources with low [O IV] flux are more frequently classified as non-masers (2 Sy2 maser and 12 Sy2 non-maser at $L_{[\mathrm{O} I V]}<$ $10^{-16} \mathrm{~W} \mathrm{~m}^{-2}$ ), while sources with high [O IV] flux are more frequently classified as masers (6 Sy2 maser and 5 Sy2 non-maser at $L_{\text {[O IV] }}>10^{-15} \mathrm{~W} \mathrm{~m}^{-2}$ ). Figure 4 displays the [O IV] fluxes of all Sy2s lying below the dividing line between obscured and unobscured sources $\left(L_{\mathrm{X}}: L_{[\mathrm{OIV}]} \approx 10\right)$. Among this subset of obscured Sy2s, the frequency of non-masers rises constantly towards lower [OIV] fluxes, in contrast to the distribution of masers. This incidence is consistent with an observational bias against the maser-detection for faint AGN. This implies that a relation exists between [O IV] flux and $\mathrm{H}_{2} \mathrm{O}$ flux, which is indeed observed in Fig. 9 and discussed in Sect. 3.5.

Because the detection of maser emission appears to be biased against sources with low flux, we conclude that among obscured sources the true fraction of masers is higher than indicated by Fig. 2.

\subsection{Extended obscuration of the dust torus and the NLR}

Figure 5 shows a histogram of the MIR $7 \mu \mathrm{m}$ continuum to [O IV] line ratio. The striking features of this diagram are:

- Sy2s populate about the same total range as Sy1s, but show a prevalence of lower $7 \mu \mathrm{m} /[\mathrm{O}$ IV] values, i.e. a $7 \mu \mathrm{m}$ 


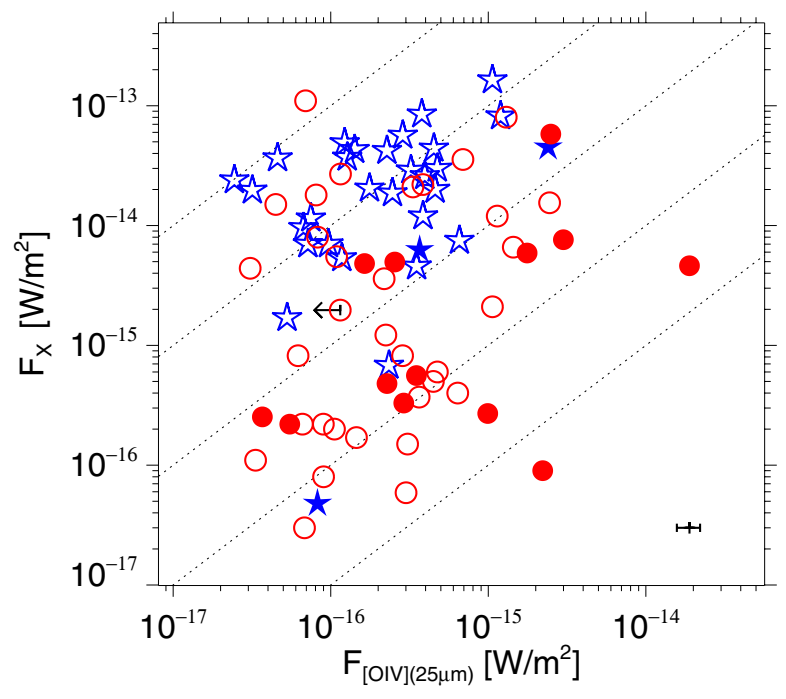

Fig. 3. Observed X-ray versus [O IV] line flux. Symbols and colors are as in Fig. 1. The dotted lines mark fixed flux ratios of 1000; 100; 10; 1; 0.1 (from top to bottom). The error bar in the lower right corner is the average relative error in all [O IV] measurements.

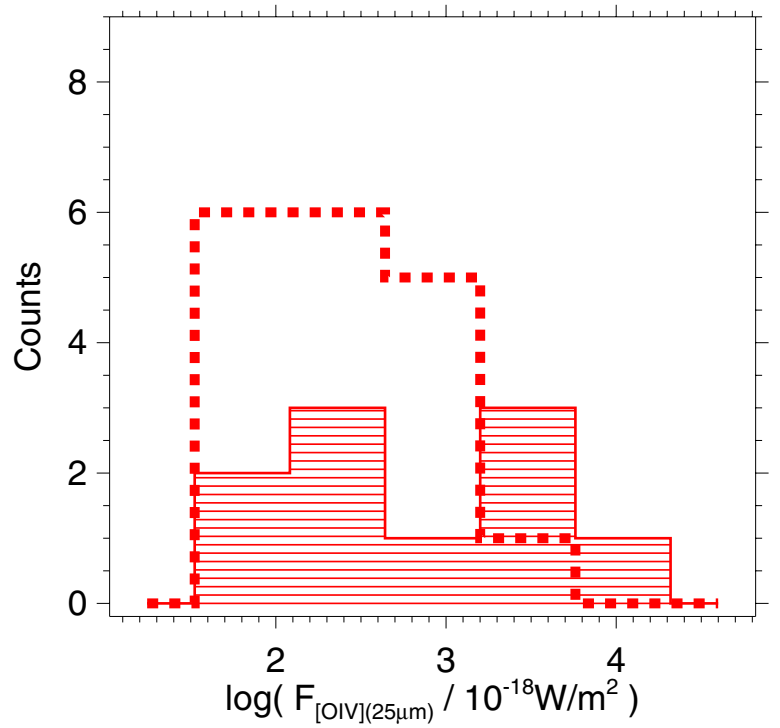

Fig. 4. [O IV] flux histogram of Sy2 masers for absorbed sources from Fig. 3 with $L_{\mathrm{X}} / L_{[\mathrm{OIV}]}<10$. Masers are represented by the shaded area, non-masers by the thick dashed histogram.

continuum deficit. On average, the ratio $7 \mu \mathrm{m} /[\mathrm{O}$ IV] of Sy $2 \mathrm{~s}$ is about a factor of three lower than that of Sy1s. This is consistent with the results obtained via radio normalization (7 $\mu \mathrm{m} / 8 \mathrm{GHz}$ ) by Buchanan et al. (2006, their Fig. 16).

- Among Sy2s, the $7 \mu \mathrm{m} /[\mathrm{O} I V]$ ratio of masers is, on average, about a factor of two lower than that of non-masers. A KS test results in a probability of $3.7 \%$ that the Sy2 masers and non-masers are drawn from the same parent distribution. Flux considerations similar to those for $L_{\mathrm{X}} / L_{[\mathrm{O} \text { IV }]}$ suggest that the true $7 \mu \mathrm{m} /[\mathrm{O} I V]$ separation of masers and non-masers will be even more pronounced once the observational bias against the detection of low flux masers is taken into account.

We assume that the deficit of the $7 \mu \mathrm{m}$ continuum in Sy2s is mainly caused by absorption of the torus dust emission. This absorption has to take place somewhere between the emitting region and the observer, hence probably in the "halo" of the torus,

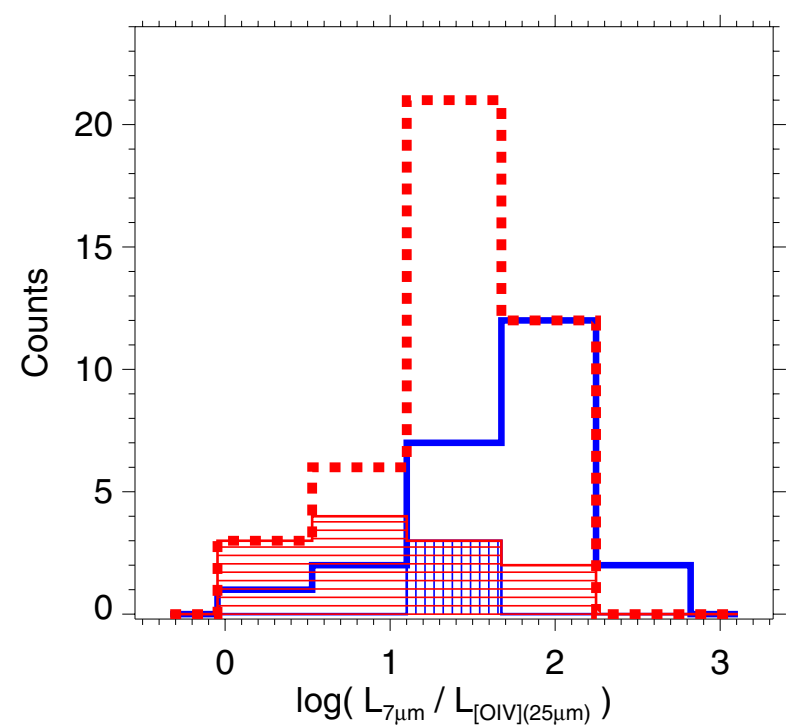

Fig. 5. Histogram of the $7 \mu \mathrm{m}$ continuum to [O IV] line ratio. Legend as in Fig. 2. All 21 upper limits from Table 3 are excluded.

i.e. in the outer part of the torus itself or in the host galaxy. It is possible that the scale height of this MIR-absorbing halo, i.e. the projected distance of absorbing material from the line-ofsight to the nuclear accretion disk, is (much) larger than the scale height of the torus itself. This is consistent with the results of a Spitzer study of CfA Seyferts (Deo et al. 2007), where sources with high $10 \mu \mathrm{m}$ silicate absorption were found to have predominantly large host-galaxy inclinations and irregularities (merger events or interactions), both of which lead to absorption through the host.

To provide additional clues on the extent of the MIRabsorbing material, we consider the [O III] $5007 \AA$ versus [OIV] line luminosity as shown in Figs. 6 and 7. The features of the $[\mathrm{OIII}] /[\mathrm{OIV}]$ distribution are similar to those of $7 \mu \mathrm{m} /[\mathrm{O}$ IV] . Most Sy2s populate the same range as Sy1s, a few Sy2s show a [O III] deficit, i.e. [O III]/[O IV] ratios lower on average about a factor 3 compared to Sy1s, consistent with the results of Baum et al. (2010) for the $12 \mu \mathrm{m}$ sample. The distribution pattern of masers and non-masers appears to be statistically indistinguishable. A KS-test results in a probability of $61 \%$ that the Sy2 masers and non-masers are drawn from the same parent population. However, the Sy2 subsample in Figs. 6 and 7 shows a distinct tail towards lower ratios, potentially caused by absorption.

The [O IV] $25.9 \mu \mathrm{m}$ line is $\sim 50$ times less affected by extinction than the optical [O III] $5007 \AA$ line. A low [O III]/[O IV] ratio argues in favor of large obscuration, as explained in Haas et al. (2005). Another explanation of the very different $[\mathrm{O} I I I] /[\mathrm{OIV}]$ ratios could be the different radiation fields in the NLR. Because the [O IV] $25.9 \mu \mathrm{m}$ line needs a higher ionization potential than the optical [O III] $5007 \AA$ line, AGN with a hard radiation field are expected to have a low ratio. The Sy2s with low $[\mathrm{O} \mathrm{III}] /[\mathrm{OIV}]$ would then be those AGN with hard radiation fields. However, this is inconsistent with other spectroscopic MIR tracers such as the [Ne II] $12.8 \mu \mathrm{m}$ to [O IV] flux ratio (Meléndez et al. 2008b). Thus, we conclude that in Figs. 6 and 7 the NLR of Sy2s with an [O III] deficit is considerably obscured.

While the [O III] obscuration may occur mainly in the innermost part of the NLR, the large extent $(>1 \mathrm{kpc})$ of the NLR suggests that the absorption is not confined to the region encircled by the dust torus. The sky-projected distribution of the 


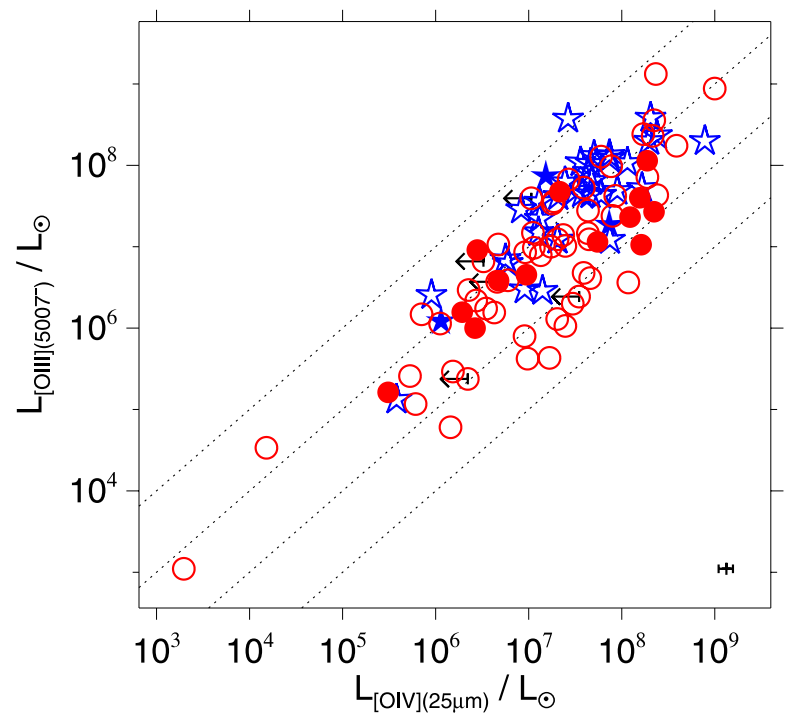

Fig. 6. Distribution of [O III] $5007 \AA$ versus [O IV] $25.9 \mu \mathrm{m}$ luminosity. Symbols are as in Fig. 1. The dotted lines mark fixed ratios 10; 1; 0.1; 0.01 (from top to bottom). The error bar in the lower right corner is the average relative error of all [O IV] measurements.

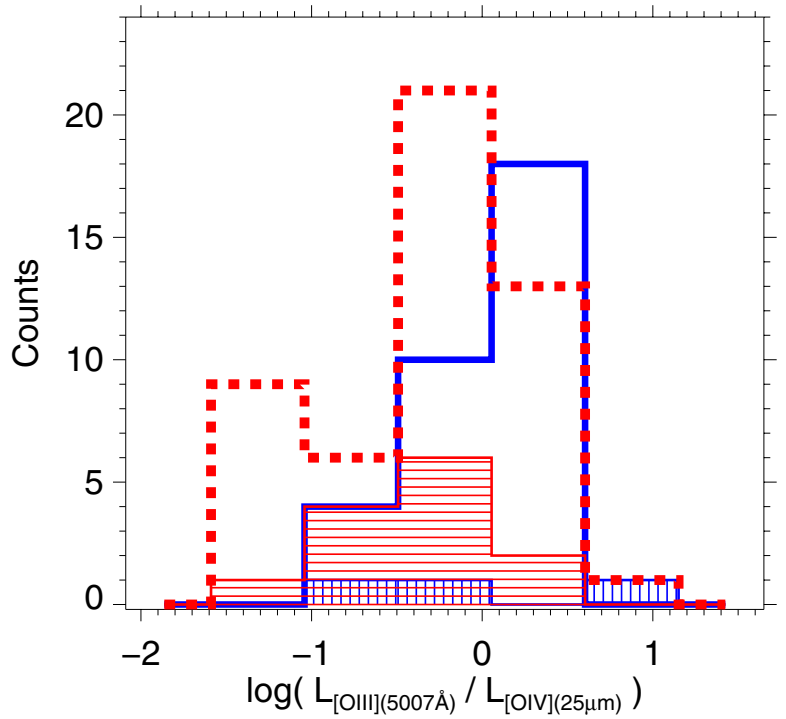

Fig. 7. Histogram of the [O III] to [O IV] ratio. Legend as in Fig. 2. Five upper limits are excluded.

absorbing material might reach further out to a considerable distance (several hundred parsec) from the line-of-sight to the nuclear accretion disk. The presence of moderately extended [O III] absorption, as well as the similarity of the $7 \mu \mathrm{m} /[\mathrm{O}$ IV] and $[\mathrm{OIII}] /[\mathrm{OIV}]$ diagrams supports the picture that the MIR absorption also takes place in a moderately extended layer, i.e. the torus halo mentioned above. Although both observables $7 \mu \mathrm{m}$ and [O III] appear to be affected by absorption in a similar fashion, we note that the distribution of $7 \mu \mathrm{m} /[\mathrm{O} \mathrm{III}]$ spans a wide range (2-700). This is unsurprising in view of the diversity of the orientation-dependent appearance of the involved emitters and absorbers even for a simple AGN model.

\subsection{Combined picture}

Why do masers and non-masers have such different distributions in X-ray/[OIV], while their distribution in $7 \mu \mathrm{m} /[\mathrm{O}$ IV] looks more similar?
Because masers need a large line-of-sight column density of velocity coherent gas, they are expected to be predominantly detected in edge-on accretion disks. Thus, the maser detection or non-detection can tell us about the disk orientation with respect to the line-of-sight. To constrain the implications in the framework of the AGN unified model, we consider two extreme cases:

1. For a disk seen edge-on, the maser is obviously most easily detected and the molecular torus is also seen more or less edge-on. In this case, the nuclear accretion disk (and its corona) is shielded by the torus, so that the X-rays are heavily obscured. If additional extended material, capable of obscuring the MIR emission, does not lie in the torus plane, the $7 \mu \mathrm{m} /[\mathrm{O} I \mathrm{~V}]$ ratio is lower.

2. For a non-maser, both the disk and torus appear to be sufficiently tilted away from edge-on, so that the nuclear X-ray absorption is relatively low. In addition, our diagrams indicate the existence of non-masers, where the torus plane is seen edge-on, although in these cases the disk might be tilted out of this plane because of the locally different angular momentum. For a non-maser, the edge-on torus also causes a high obscuration of the X-ray nucleus. On the other hand, irrespective of the disk and torus orientation, the MIR continuum can be absorbed or not depending on the line-of-sight through the extended host.

From these two extreme cases, we see the following. While the requirement for heavy absorption of the nuclear X-rays is that the line-of-sight has to hit a rather compact area of very high column density, the area of the torus emission and even more so the area of the (bright) NLR emission is orders of magnitude larger, so that the absorber must also cover a larger area. If the absorption of X-rays and $7 \mu \mathrm{m}$ occurs on different spatial scales, the strength of the obscuration in each wavelength range may be sensitive to small differences in the aspect angle. Furthermore, because the MIR-absorbing material is located farther away from the line-of-sight to the nucleus, it is less reliable for predicting whether a maser will be detected.

\subsection{Comparison of the three samples with other known masers}

Our combined sample was compiled from three complete samples with good coverage in the Spitzer archive. Table 1 shows the overlaps between the samples. We note that each sample is incomplete because of the limited availability of data in the Spitzer IRS archive and of X-ray and [O III] measurements in the literature (see Table 2). Moreover, maser surveys have not been performed with homogeneous properties (sensitivity, velocity coverage) nor carried out for all sources of our combined sample.

The three samples are based on different selection criteria: Optically selected Seyferts in the CfA sample and IRAS selected sources in the $12 \mu \mathrm{m}$ and F25/F60 sample. Thus, it is possible that they are affected by different biases with respect to potential maser detection. The fraction of Sy2 masers to non-masers increases from $1 / 4(4 / 16)$ in the CfA sample, to $\sim 1 / 3(11 / 29)$ in the $12 \mu \mathrm{m}$ sample and to $\gtrsim 1(8 / 6)$ in the IRAS F25/F60 sample. This is consistent with the well-known finding that the mid- and far-infrared wavelengths select more obscured AGN than the optical bands.

However, the range of luminosity ratios $\left(L_{\mathrm{X}} / L_{[\mathrm{O} I V]}\right.$, $\left.L_{7 \mu \mathrm{m}} / L_{[\mathrm{O} \text { IV }]}, L_{[\mathrm{O} \text { III }]} / L_{[\mathrm{O} \text { IV }]}\right)$, listed in Table 2 , are similar for all three samples. This also indicates that among optical selected masing sources, some can be obscured at a level similar to that of 


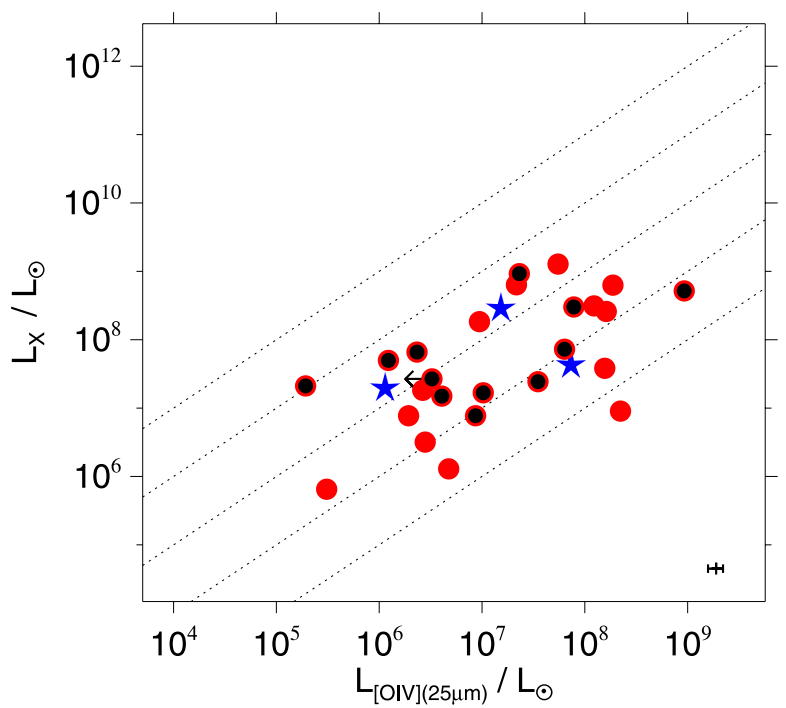

Fig. 8. Observed 2-10 keV X-rays plotted against [O IV] line luminosity. Sy 1 masers are represented by blue stars and Sy2 masers by red dots. Off-sample Sy2 masers are marked with black dots. The dotted lines mark fixed flux ratios of $1000 ; 100 ; 10 ; 1 ; 0.1$ (from top to bottom). The error bar in the lower right corner is the average relative error of all [O IV] measurements.

infrared selected sources ${ }^{5}$. To summarize, the result of all three samples (CfA, $12 \mu \mathrm{m}$, and IRAS F25/F60) are similar in that they point consistently to a prevalence of maser detections in Sy2s with high X-ray obscuration and one may expect that this also holds in general for Seyfert galaxies.

Are our selected Sy2 masers representative of all 52 known Sy2 masers (Zhang et al. 2010)? To address this question, we compare our in-sample Sy2 masers with all remaining 37 offsample Sy2 masers.

In Fig. 8, we compare X-ray and [O IV] luminosities between in- and off-sample masers. The comparison refers to those masers with X-ray and [O IV] fluxes available, i.e. 12 off-sample Sy2s, 13 in-sample Sy2s, and 3 in-sample Sy1s. Both the insample and off-sample roughly populate the same $L_{\mathrm{X}} / L_{[\mathrm{O} \text { IV] }}$ range. However, the $L_{\mathrm{X}} / L_{[\mathrm{O} I \mathrm{IV}]}$ ratio is, on average, about a factor of two higher for the off-sample than the in-sample masers. This indicates that the off-sample Sy 2 masers may be less absorbed than the in-sample ones. Compared with the in-sample Sy2 nonmasers (omitted in Fig. 8, see Fig. 1), however, the off-sample masers show, on average, about a factor of two lower $L_{\mathrm{X}} / L_{[\mathrm{O} I \mathrm{~V}]}$, hence considerably high obscuration.

Some off-sample masers exhibit spurious flux ratios indicative of no obscuration (i.e. $L_{\mathrm{X}} / L_{[\mathrm{O} I V]}>10$ for 4 objects). Among them, we find two nearby extended sources, NGC 4258 and NGC 4945, in which the X-ray emission was associated with star formation by Risaliti (2002) and Strickland et al. (2004).

To summarize, the large overlap and the lack of significant differences between in- and off-sample Sy 2 masers indicates that the results for our combined sample of Sy2 masers can be extended to all known Sy2 masers.

We note that the inhomogeneous selection of all off-sample masers and non-masers precludes any meaningful comparison of maser to non-maser statistics with our in-sample data. A KS test shows a probability of $63 \%$ that subsets of both, off- and insample masers, are drawn from the same parent distribution and

\footnotetext{
${ }^{5}$ Even for infrared-selected AGN, optical criteria influence the sample, because the Seyfert identification is done using optical spectroscopy.
}

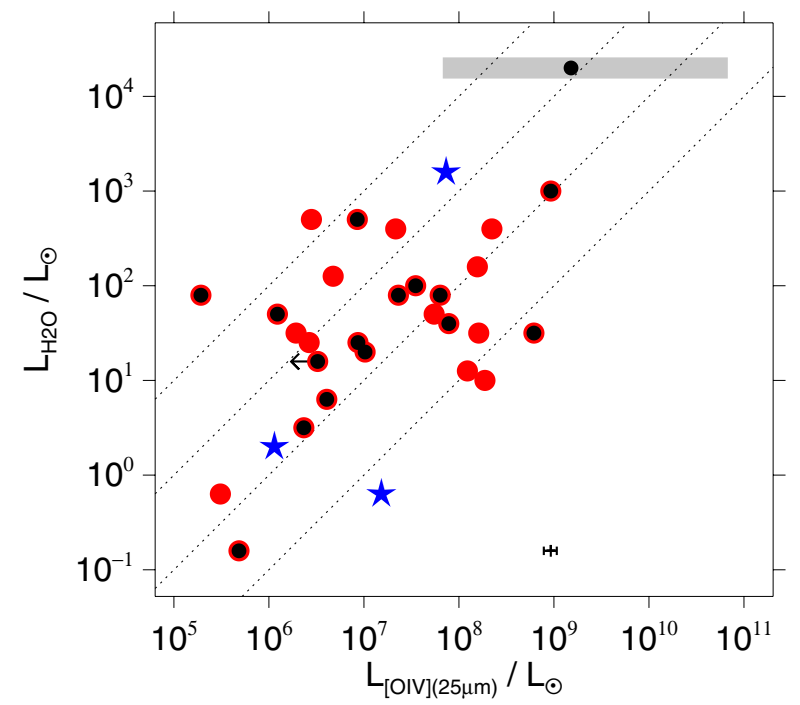

Fig. 9. Maser $\mathrm{H}_{2} \mathrm{O}$ versus [O IV] luminosity. Symbols and colors are as in Fig. 8. The dotted lines mark fixed ratios $10^{-4} ; 10^{-5} ; 10^{-6} ; 10^{-7}$ (from top to bottom). For comparison, the expected position of the $z=0.66$ maser SDSSJ0804+3607 is marked with a black dot and a gray bar covering the range $0.1 \times L_{[\mathrm{OIII}]}<L_{[\mathrm{OIV}]}<100 \times L_{[\mathrm{O} \text { III] }}$ assumed from Fig. 6.

the above-mentioned difference in $L_{\mathrm{X}} / L_{[\mathrm{O} I \mathrm{IV}]}$ is only by chance. However, a KS test between the in-sample non-masers and the off-sample masers similarly yields a $60 \%$ probability that they are drawn from the same parent distribution. Comparing the $L_{\mathrm{X}} / L_{\text {[O IV] }}$ ratio of the in-sample Sy1 non-masers with those of the off-sample Sy 2 masers, shows a probability of $0.16 \%$ of their being drawn from the same parent distribution. This indicates that the off-sample Sy2 masers differ significantly from the unobscured Sy1 non-maser.

\subsection{Maser and AGN luminosity}

A search for $\mathrm{H}_{2} \mathrm{O}$ masers in 274 high-redshift $(0.3<z<0.8)$ SDSS type-2 AGN, of which half are type-2 quasars (Bennert et al. 2009) found only one maser (SDSSJ0804+3607, Barvainis $\&$ Antonucci 2005). The high rate of non-detections in these luminous AGN might be due to limited observational sensitivity or intrinsic differences between low- and high-luminosity AGN. These differences could for instance be that in a high-luminosity AGN the accretion disk becomes hotter so that the density required for maser emission falls below a critical limit. If this were frequently the case, one would expect a relative decline in $\mathrm{H}_{2} \mathrm{O}$ maser luminosity with increasing AGN luminosity. On the other hand, the SDSS $\mathrm{H}_{2} \mathrm{O}$ maser survey was relatively shallow, because one was interested in finding masers that are sufficiently bright for spatially resolved follow-up VLBI observations.

We consider how far the Seyfert sample can help us to distinguish between these two possibilities (i.e. by investigating whether or not $L_{\mathrm{H} 2 \mathrm{O}} / L_{[\mathrm{O} I V]}$ declines with increasing $L_{[\mathrm{OIV}]}$ ). A remarkable feature of Fig. 1 is that maser-detections and non-detections are quite evenly distributed across the whole [OIV] luminosity range covering about four orders of magnitude. Thus, our data do not indicate a trend where the frequency of non-masers increases with luminosity. We have also seen that the available maser observations of the Seyferts are biased against maser detection in faint (and distant) AGN (Fig. 4).

Figure 9 shows the (isotropic) maser luminosity versus the AGN luminosity traced by [OIV]. In addition to the Seyfert sample, we have plotted the expected position of 
SDSSJ0804+3607 at $z=0.66$, the only QSO-2 maser detection. Because this source had not been observed with Spitzer IRS, we derived $L_{[\mathrm{OIV}]}$ from $L_{[\mathrm{O} \text { III] }}$ using the range $0.1 \times L_{[\mathrm{O} \text { III] }}<$ $L_{[\mathrm{OIV}]}<100 \times L_{\text {[O III] }}$ as indicated in Fig. 6, which is also valid for higher luminosity AGN (Haas et al. 2005). At a given [OIV] luminosity, the maser luminosity spreads over three orders of magnitude (Fig. 9). One explanation for the large spread is that the maser emission is not isotropic, and hence the derived maser luminosity depends sensitively on the maser direction with respect to the line-of-sight. The Seyfert sample alone indicates only a marginal correlation in Fig. 9, with a Pearson correlation coefficient of 0.32 for all Sy 2 masers of our combined sample that is not significant at the 5\% level. Adding the offsample Sy 2 masers changes the coefficient to 0.46 which would then be significant, but this correlation might also be an artifact of distance in the luminosities. However, in addition to the position of SDSSJ0804+3607 and that its assumed $\mathrm{H}_{2} \mathrm{O} /[\mathrm{O}$ IV] ratio lies in the same range as the lower luminosity AGN, argues in favor of a physical connection between maser and AGN luminosity.

The numerous non-masers among the SDSS QSO-2s (Bennert et al. 2009) have a [O III] luminosity similar to that of SDSSJ0804+3607, hence are expected to populate a similar $L_{[\mathrm{O} I V]}$ range in Fig. 9. The $\mathrm{H}_{2} \mathrm{O}$ maser upper limits ${ }^{6}$, found for these QSO-2s by Bennert et al. (2009), lie even above J0804+3607. Thus, the upper limits are not stringent enough to support a relative decline of maser luminosity with increasing AGN luminosity. This, together with the sufficiently high $L_{\mathrm{H} 2 \mathrm{O}} / L_{[\mathrm{O} \text { IV] }}$ ratio of $\mathrm{J} 0804+3607$, leads us to conclude that the main reason for the high rate of maser non-detections is insufficient observational sensitivity, rather than basic differences between low-and high-luminosity AGN for hosting a maser.

\section{Conclusion}

To understand the connection between $\mathrm{H}_{2} \mathrm{O}$ maser detection rate and nuclear extinction, we have used the $[\mathrm{OIV}]_{25.9} \mu \mathrm{m}$ line and the $7 \mu \mathrm{m}$ continuum flux from Spitzer spectra of a wellselected sample of 114 Seyfert galaxies from the CfA, $12 \mu \mathrm{m}$, and IRAS F25/F60 catalogs, for which a maser search has been performed. These data have been compared to hard X-ray and [O III] $5007 \AA$ fluxes from the literature. We have analyzed the data in the framework of the orientation-dependent AGN unified scheme, yielding the following results:

1. Comparing hard X-rays to [O IV] flux, Sy2s exhibit, on average, an about ten times lower X-ray to [OIV] ratio than Sy1s. Masers reside primarily in X-ray absorbed sources (i.e. those with low $L_{X} / L_{[O I V]}$ ratios). Sy 2 masers have on average about four times less X-ray flux normalized by [OIV] than non-maser Sy2s. This is consistent with the geometric alignment of both the X-ray absorber and the maser emitting region in the accretion disk. Non-masers do not tend to reside predominantly in strongly absorbed sources. However, our data uncover an observational bias against faint sources, in the sense that more sensitive maser observations might reveal more absorbed sources to house a maser.

2. For the $7 \mu \mathrm{m}$ to [OIV] flux ratio, we have found that most Sy2s are spread across the same range as Sy1s. However, some sources have a significantly lower ratio, rendering the Sy2s on average about three times lower than Sy1s. These cases can be explained by an extended dusty absorber covering the $7 \mu \mathrm{m}$ emitting torus region. Maser detections also

$6 \frac{L_{\mathrm{H} 2 O}}{L_{\odot}}=0.0039 \times \frac{1}{1+z} \times\left(\frac{D_{\mathrm{L}}}{\mathrm{Mpc}}\right)^{2}$. appear to correspond to $7 \mu \mathrm{m}$ absorbed sources, but with less significance than in the X-ray to [O IV] comparison. This suggests that the geometric alignment of the MIR absorber with the maser emitting disk is not as perfect as the supposed alignment of the disk with the X-ray absorber.

3. The [O III] to [O IV] flux ratio also indicates the presence of extended obscuration in some Sy2s that blocks the optical emission from the NLR. Masers and non-masers are distributed very similarly in the [O III] to [O IV] plot. The fraction of maser-detections is not significantly higher for sources with this extended absorption. This leads us to conclude that the matter distribution for the [O III] absorber is not essential for the prediction of a maser detection. Moreover, it is possible that a substantial fraction of the absorption of AGN emission could occur in extended regions outside the torus that are not necessarily aligned with the torus or AGN sub-structure.

4. The separate three samples, CfA, $12 \mu \mathrm{m}$, and IRAS F25/F60 provide very similar results. The Sy2 maser to non-maser fraction increases from optical to infrared selection. The Sy2 masers of our combined sample have a similar range of $L_{[\mathrm{OIV}]}$ and $L_{\mathrm{H} 2 \mathrm{O}}$ as the known off-sample Sy2 masers. While, on average, the off-sample masers are a factor of two less obscured, as inferred by the $L_{\mathrm{X}} / L_{\text {[O IV] }}$ ratio, they are still considerably obscured compared to Sy $1 \mathrm{~s}$ from our combined sample. Thus, the results obtained for our combined sample of Sy2 masers may also hold for all remaining Sy2 maser sources that have no Spitzer or X-ray data available.

5. After supplementing our combined sample with the remaining known Sy2 masers that were not included in it the $\mathrm{H}_{2} \mathrm{O}$ maser luminosity appears to be correlated with the AGN luminosity traced by [OIV], although it has a large spread. We do not find any evidence of physical differences between low-and high-luminosity AGN hosting a maser.

The results demonstrate that heavy X-ray absorption is an indicator of a high probability of detecting a maser. The $7 \mu \mathrm{m}$ absorption can also be used to find maser candidates, but with a lower probability.

Acknowledgements. The work is based on observations made with the Spitzer Space Telescope, which is operated by the Jet Propulsion Laboratory, California Institute of Technology under a contract with NASA. This research has made use of the NASA/IPAC Extragalactic Database (NED) which is operated by the Jet Propulsion Laboratory, California Institute of Technology, under contract with the National Aeronautics and Space Administration. This publication is supported as a project of the Nordrhein-Westfälische Akademie der Wissenschaften und der Künste in the framework of the academy program by the Federal Republic of Germany and the state Nordrhein-Westfalen. We thank the referee J. S. Zhang for his careful review of the manuscript.

\section{References}

Alonso-Herrero, A., Ward, M. J., \& Kotilainen, J. K. 1997, MNRAS, 288, 977 Antonucci, R. 1993, ARA\&A, 31, 473

Awaki, H., Ueno, S., Taniguchi, Y., \& Weaver, K. A. 2000, ApJ, 542, 175

Baker, J. C. 1997, MNRAS, 286, 23

Barvainis, R., \& Antonucci, R. 2005, ApJ, 628, L89

Bassani, L., Dadina, M., Maiolino, R., et al. 1999, ApJS, 121, 473

Baum, S. A., Gallimore, J. F., O’Dea, C. P., et al. 2010, ApJ, 710, 289

Becker, R., Henkel, C., Wilson, T. L., \& Wouterloot, J. G. A. 1993, A\&A, 268 483

Bennert, N., Falcke, H., Schulz, H., Wilson, A. S., \& Wills, B. J. 2002, ApJ, 574, L105

Bennert, N., Jungwiert, B., Komossa, S., Haas, M., \& Chini, R. 2006, A\&A, 446 919

Bennert, N., Barvainis, R., Henkel, C., \& Antonucci, R. 2009, ApJ, 695, 276

Bianchi, S., Balestra, I., Matt, G., Guainazzi, M., \& Perola, G. C. 2003, A\&A, 402,141

Bianchi, S., Miniutti, G., Fabian, A. C., \& Iwasawa, K. 2005, MNRAS, 360, 380 
Blustin, A. J., Branduardi-Raymont, G., Behar, E., et al. 2002, A\&A, 392, 453 Boroson, T. A., \& Meyers, K. A. 1992, ApJ, 397, 442

Braatz, J. 2008, in A Decade of Dark Energy

Braatz, J. A., \& Gugliucci, N. E. 2008, ApJ, 678, 96

Braatz, J. A., Wilson, A. S., \& Henkel, C. 1996, ApJS, 106, 51

Braatz, J. A., Wilson, A. S., \& Henkel, C. 1997, ApJS, 110, 321

Braatz, J. A., Wilson, A. S., Henkel, C., Gough, R., \& Sinclair, M. 2003, ApJS, 146, 249

Braatz, J. A., Henkel, C., Greenhill, L. J., Moran, J. M., \& Wilson, A. S. 2004, ApJ, 617, L29

Braito, V., Franceschini, A., Della Ceca, R., et al. 2003, A\&A, 398, 107

Buchanan, C. L., Gallimore, J. F., O’Dea, C. P., et al. 2006, AJ, 132, 401

Cappi, M., Panessa, F., Bassani, L., et al. 2006, A\&A, 446, 459

Claussen, M. J., \& Lo, K. 1986, ApJ, 308, 592

Dahari, O., \& De Robertis, M. M. 1988, ApJS, 67, 249

de Grijp, M. H. K., Keel, W. C., Miley, G. K., Goudfrooij, P., \& Lub, J. 1992, A\&AS, 96, 389

Deo, R. P., Crenshaw, D. M., Kraemer, S. B., et al. 2007, ApJ, 671, 124

Deo, R. P., Richards, G. T., Crenshaw, D. M., \& Kraemer, S. B. 2009, ApJ, 705,

di Serego Alighieri, S., Cimatti, A., Fosbury, R. A. E., \& Hes, R. 1997, A\&A, 328,510

Done, C., Madejski, G. M., Życki, P. T., \& Greenhill, L. J. 2003, ApJ, 588, 763

Elitzur, M. 2002, in Cosmic Masers: From Proto-Stars to Black Holes, ed V. Migenes, \& M. J. Reid, IAU Symp., 206, 452

Genzel, R., Lutz, D., Sturm, E., et al. 1998, ApJ, 498, 579

Gondoin, P., Barr, P., Lumb, D., et al. 2001, A\&A, 378, 806

Gondoin, P., Orr, A., Lumb, D., \& Santos-Lleo, M. 2002, A\&A, 388, 74

Gondoin, P., Orr, A., \& Lumb, D. 2003a, A\&A, 398, 967

Gondoin, P., Orr, A., Lumb, D., \& Siddiqui, H. 2003b, A\&A, 397, 883

González-Martín, O., Masegosa, J., Márquez, I., Guerrero, M. A., \& DultzinHacyan, D. 2006, A\&A, 460, 45

Greenhill, L. J., Moran, J. M., Reid, M. J., et al. 1990, ApJ, 364, 513

Greenhill, L. J., Moran, J. M., Reid, M. J., Menten, K. M., \& Hirabayashi, H. 1993, ApJ, 406, 482

Greenhill, L. J., Henkel, C., Becker, R., Wilson, T. L., \& Wouterloot, J. G. A 1995, A\&A, 304, 21

Greenhill, L. J., Herrnstein, J. R., Moran, J. M., Menten, K. M., \& Velusamy, T. 1997, ApJ, 486, L15

Greenhill, L. J., Ellingsen, S. P., Norris, R. P., et al. 2002, ApJ, 565, 836

Greenhill, L. J., Tilak, A., \& Madejski, G. 2008, ApJ, 686, L13

Gu, Q., Melnick, J., Fernandes, R. C., et al. 2006, VizieR Online Data Catalog, 736,60480

Guainazzi, M., Matt, G., \& Perola, G. C. 2005, A\&A, 444, 119

Haas, M., Siebenmorgen, R., Schulz, B., Krügel, E., \& Chini, R. 2005, A\&A, 442, L39

Hagiwara, Y., Diamond, P. J., \& Miyoshi, M. 2002, A\&A, 383, 65

Hagiwara, Y., Diamond, P. J., Miyoshi, M., Rovilos, E., \& Baan, W. 2003, MNRAS, 344, L53

Haschick, A. D., \& Baan, W. A. 1985, Nature, 314, 144

Heckman, T. M., Crane, P. C., \& Balick, B. 1980, A\&AS, 40, 295

Heckman, T. M., Ptak, A., Hornschemeier, A., \& Kauffmann, G. 2005, ApJ, 634, 161

Henkel, C. 2008, in A Decade of Dark Energy

Henkel, C., Guesten, R., Downes, D., et al. 1984, A\&A, 141, L1

Henkel, C., Wouterloot, J. G. A., \& Bally, J. 1986, A\&A, 155, 193

Henkel, C., Wang, Y. P., Falcke, H., Wilson, A. S., \& Braatz, J. A. 1998, A\&A 335,463

Henkel, C., Peck, A. B., Tarchi, A., et al. 2005, A\&A, 436, 75

Herrnstein, J. R., Moran, J. M., Greenhill, L. J., et al. 1999, Nature, 400, 539

Hes, R., Barthel, P. D., \& Fosbury, R. A. E. 1996, A\&A, 313, 423

Ho, L. C. 2005, ApJ, 629, 680

Ho, L. C., Filippenko, A. V., \& Sargent, W. L. 1995, ApJS, 98, 477

Ho, L. C., Filippenko, A. V., \& Sargent, W. L. W. 1997, ApJS, 112, 315

Holczer, T., Behar, E., \& Kaspi, S. 2007, ApJ, 663, 799

Huchra, J., \& Burg, R. 1992, ApJ, 393, 90

Immler, S., Brandt, W. N., Vignali, C., et al. 2003, AJ, 126, 153

Iyomoto, N., Makishima, K., Fukazawa, Y., et al. 1996, PASJ, 48, 231

Kewley, L. J., Heisler, C. A., Dopita, M. A., \& Lumsden, S. 2001, ApJS, 132,

Kim, D., Sanders, D. B., Veilleux, S., Mazzarella, J. M., \& Soifer, B. T. 1995, ApJS, 98, 129

Kondratko, P. T., Greenhill, L. J., \& Moran, J. M. 2006a, ApJ, 652, 136

Kondratko, P. T., Greenhill, L. J., Moran, J. M., et al. 2006b, ApJ, 649, 561

Lamastra, A., Bianchi, S., Matt, G., et al. 2009, A\&A, 504, 73

Lawson, A. J., \& Turner, M. J. L. 1997, MNRAS, 288, 920

Levenson, N. A., Heckman, T. M., Krolik, J. H., Weaver, K. A., \& Życki, P. T. 2006, ApJ, 648, 111
Lo, K. Y. 2005, ARA\&A, 43, 625

Lumsden, S. L., \& Alexander, D. M. 2001, MNRAS, 328, L32

Lumsden, S. L., Alexander, D. M., \& Hough, J. H. 2004, MNRAS, 348, 1451

Maiolino, R., \& Rieke, G. H. 1995, ApJ, 454, 95

Malizia, A., Landi, R., Bassani, L., et al. 2007, ApJ, 668, 81

Matsumoto, C., Nava, A., Maddox, L. A., et al. 2004, ApJ, 617, 930

Matt, G., Perola, G. C., Fiore, F., et al. 2000, A\&A, 363, 863

Meléndez, M., Kraemer, S. B., Armentrout, B. K., et al. 2008a, ApJ, 682, 94

Meléndez, M., Kraemer, S. B., Schmitt, H. R., et al. 2008b, ApJ, 689, 95

Miniutti, G., Ponti, G., Dadina, M., Cappi, M., \& Malaguti, G. 2007, MNRAS, 375,227

Moustakas, J., \& Kennicutt, Jr., R. C. 2006, ApJS, 164, 81

Mulchaey, J. S., Koratkar, A., Ward, M. J., et al. 1994, ApJ, 436, 586

Nakai, N., Inoue, M., Miyazawa, K., Miyoshi, M., \& Hall, P. 1995, PASJ, 47, 771

Nandra, K., O’Neill, P. M., George, I. M., \& Reeves, J. N. 2007, MNRAS, 382,

Nenkova, M., Sirocky, M. M., Ivezić, Ž., \& Elitzur, M. 2008, ApJ, 685, 147

Netzer, H., Mainieri, V., Rosati, P., \& Trakhtenbrot, B. 2006, A\&A, 453, 525

Oliva, E., Salvati, M., Moorwood, A. F. M., \& Marconi, A. 1994, A\&A, 288, 457

Panessa, F., Bassani, L., Cappi, M., et al. 2006, A\&A, 455, 173

Perola, G. C., Matt, G., Fiore, F., et al. 2000, A\&A, 358, 117

Perola, G. C., Matt, G., Cappi, M., et al. 2002, A\&A, 389, 802

Phillips, M. M., Charles, P. A., \& Baldwin, J. A. 1983, ApJ, 266, 485

Polletta, M., Bassani, L., Malaguti, G., Palumbo, G. G. C., \& Caroli, E. 1996, ApJS, 106, 399

Ramolla, M. 2009, Middle-Infrared Spectroscopy of Seyfert Galaxies, Diploma Thesis, Ruhr-Universität Bochum

Rao, A. R., Singh, K. P., \& Vahia, M. N. 1992, MNRAS, 255, 197

Reeves, J. N., \& Turner, M. J. L. 2000, MNRAS, 316, 234

Reynolds, C. S. 1997, MNRAS, 286, 513

Risaliti, G. 2002, A\&A, 386, 379

Risaliti, G., Gilli, R., Maiolino, R., \& Salvati, M. 2000, A\&A, 357, 13

Rush, B., \& Malkan, M. A. 1993, in BAAS, 25, 1362

Sato, N., Yamauchi, A., Ishihara, Y., et al. 2005, PASJ, 57, 587

Schmitt, H. R., Donley, J. L., Antonucci, R. R. J., Hutchings, J. B., \& Kinney,

A. L. 2003, ApJS, 148, 327

Schweitzer, M., Lutz, D., Sturm, E., et al. 2006, ApJ, 649, 79

Severgnini, P., Risaliti, G., Marconi, A., Maiolino, R., \& Salvati, M. 2001, A\&A, 368,44

Shang, Z., Wills, B. J., Wills, D., \& Brotherton, M. S. 2007, AJ, 134, 294

Sharples, R. M., Longmore, A. J., Hawarden, T. G., \& Carter, D. 1984, MNRAS, 208, 15

Shinozaki, K., Miyaji, T., Ishisaki, Y., Ueda, Y., \& Ogasaka, Y. 2006, AJ, 131, 2843

Shu, X. W., Wang, J. X., Jiang, P., Fan, L. L., \& Wang, T. G. 2007, ApJ, 657, 167

Smith, D. A., \& Wilson, A. S. 2001, ApJ, 557, 180

Smith, J. D. T., \& Draine, B. T. 2008, PAHFIT web description,

http://tir.astro.utoledo.edu/jdsmith/research/pahfit.php

Smith, J. D. T., Draine, B. T., Dale, D. A., et al. 2007, ApJ, 656, 770

Spinoglio, L., \& Malkan, M. A. 1989, ApJ, 342, 83

Storchi-Bergmann, T., Kinney, A. L., \& Challis, P. 1995, ApJS, 98, 103

Strickland, D. K., Heckman, T. M., Colbert, E. J. M., Hoopes, C. G., \& Weaver, K. A. 2004, ApJS, 151, 193

Terashima, Y., Iyomoto, N., Ho, L. C., \& Ptak, A. F. 2002, ApJS, 139, 1

Tilak, A., Greenhill, L. J., Done, C., \& Madejski, G. 2008, ApJ, 678, 701

Tommasin, S., Spinoglio, L., Malkan, M. A., \& Fazio, G. 2010, ApJ, 709, 1257

Tran, H. D. 2003a, ApJ, 583, 632

Tran, H. T. 2003b, New Astron. Rev., 47, 1091

Turner, T. J., \& Pounds, K. A. 1989, MNRAS, 240, 833

Turner, T. J., George, I. M., Nandra, K., \& Mushotzky, R. F. 1997, ApJS, 113, 23

Ueda, Y., Ishisaki, Y., Takahashi, T., Makishima, K., \& Ohashi, T. 2005, ApJS 161,185

Ueno, S., Ward, M. J., O’Brien, P. T., Stirpe, G. M., \& Matt, G. 2000, Adv. Space Res., 25, 823

Vaceli, M. S., Viegas, S. M., Gruenwald, R., \& de Souza, R. E. 1997, AJ, 114, 1345

Véron-Cetty, M., \& Véron, P. 2006, A\&A, 455, 773

Verrecchia, F., in't Zand, J. J. M., Giommi, P., et al. 2007, A\&A, 472, 705

Whittle, M. 1992, ApJS, 79, 49

Whittle, M., \& Wilson, A. S. 2004, AJ, 127, 606

Zhang, J. S., \& Fan, J. H. 2009, Sci. Chin., 52, G6, 960

Zhang, J. S., Henkel, C., Kadler, M., et al. 2006, A\&A, 450, 933

Zhang, J. S., Henkel, C., Guo, Q., Wang, H. G., \& Fan, J. H. 2010, ApJ, 708, 1528 